\title{
Searching for a good model for systemic sclerosis: the molecular profile and vascular changes occurring in UCD-200 chickens strongly resemble the early phase of human systemic sclerosis
}

\author{
Paola Cipriani ${ }^{1}$, Paola Di Benedetto ${ }^{1}$, Hermann Dietrich ${ }^{2}$, Piero Ruscitti ${ }^{1}$, Vasiliki Liakouli ${ }^{1}$, \\ Francesco Carubbi ${ }^{1}$, Ilenia Pantano ${ }^{1}$, Onorina Berardicurti ${ }^{1}$, Roswitha Sgonc ${ }^{3}$, Roberto Giacomelli ${ }^{1}$
}

\begin{abstract}
1Department of Applied Clinical Sciences and Biotechnology, Rheumatology Unit, School of Medicine, University of L'Aquila, L'Aquila, Italy

${ }^{2}$ Central Laboratory Animal Facilities, Innsbruck Medical University, Innsbruck, Austria ${ }^{3}$ Division of Experimental Pathophysiology and Immunology, Biocenter, Innsbruck Medical University, Innsbruck, Austria
\end{abstract}

Submitted: 27 August 2014

Accepted: 2 December 2014

Arch Med Sci 2016; 12, 4: 828-843

DOI: 10.5114 /aoms.2016.60970

Copyright (c) 2016 termedia \& Banach

\section{Abstract}

Introduction: Vascular injury and endothelial cell (EC) apoptosis are the earliest events in systemic sclerosis (SSC), before the onset of fibrosis, and stromal cell-derived factor 1 (SDF-1), vascular endothelial growth factor (VEGFA), endothelin-1 (ET-1) and platelet-derived growth factors (PDGF-BB) represent the key molecules to study the link between vascular injury and fibrosis during SSC. The University of California at Davis line 200 (UCD-200) chickens display the same hallmarks of human SSc: vascular occlusion, perivascular lymphocytic infiltration and fibrosis of skin and internal organs. In this study we assessed both cytokines and growth factors involved in the early phases of the UCD-200 chickens' skin lesions, to determine whether these animals might represent an appropriate experimental model to study the pathogenesis of SSc.

Material and methods: Immunofluorescence analysis was performed on human SSC skin, human healthy control (hHC) skin, UCD-200 combs and HC H.B15 chicken (CHC) combs, using anti-SDF-1, CXCR4, VEGFA, VEGF receptor 1 (VEGFR1), VEGF receptor 2 (VEGFR2), ET-1, ET receptor A (ETAR), ET receptor $B$ (ETBR), PDGF-BB, and PDGF receptor (PDGFR) antibodies. The plasma concentrations of SDF-1, VEGFA, ET-1 and PDGF-BB were determined by ELISA.

Results: All the molecules analyzed showed higher levels in SSc patients and UCD-200 chickens than in hHC and CHC. Furthermore, the levels of the assessed molecules paralleled the severity of comb involvement.

Conclusions: The molecular similarities between avian and human SSc, observed in this study, suggest that the UCD-200 chickens are an interesting model for translational approaches to SSC.

Key words: angiogenesis, fibrosis, animal model.

\section{Introduction}

Systemic sclerosis (SSc) is an autoimmune disease of unknown etiology, affecting different organs. The disease is characterized by fibroproliferative vasculopathy, tissue fibrosis and activation of the immune system [1].

\author{
Corresponding author: \\ Paola Cipriani \\ Department \\ of Applied Clinical \\ Sciences and Biotechnology \\ Rheumatology Unit \\ School of Medicine \\ University of L'Aquila \\ Delta 6 Building \\ Via dell'Ospedale \\ 67100 L'Aquila, Italy \\ Phone: +390862434775 \\ E-mail: paola.cipriani@ \\ cc.univaq.it
}


The vasculopathy occurs early in the disease and precedes the onset of fibrosis. The vascular damage is the primary event leading to progressive capillary loss. However, despite the severe hypoxia and elevated levels of pro-angiogenic molecules, adaptive angiogenesis is largely defective in SSc. Endothelial cell (ECS) and pericytes (PS) are the main players of formation, remodeling and maintenance of the vascular bed. During SSc, impaired interplay between ECs and Ps might induce a perturbation of vascular repair, leading to a phenotypic switch of Ps toward myofibroblasts [2]. Several molecules are involved in the control and modulation of P/EC interactions, such as stromal cell-derived factor 1 (SDF-1), vascular endothelial growth factor (VEGF), tumor growth factor (TGF)- $\beta 1$, endothelin-1 (ET-1) and platelet-derived growth factors (PDGF-BB), and all of these may be considered as key molecules to study the link between vascular injury and fibrosis, during SSc [3].

Although in the last years many of these mechanisms have been evaluated, little is known about the earlier pathogenetic steps of SSc, triggering the transition from vascular damage to a fibrotic process, and a translational approach is strongly needed to address this topic.

UCD-200 chicken is the only animal model displaying all the hallmarks of human SSc, i.e. vascular occlusion, severe perivascular lymphocytic infiltration of skin and viscera, fibrosis of skin and internal organs, antinuclear antibodies, and distal polyarthritis [4, 5]. These chickens spontaneously develop an inherited scleroderma-like disease with an initial inflammatory stage, $1-3$ weeks after hatching, prominently in the comb, evolving to fibrosis with accumulation of types I, III, and VI collagen, in the affected tissues. Alterations start in the skin within the first week after hatching and then rapidly extend to internal organs, and $90 \%$ of the birds are afflicted at the age of 5 weeks [6].

In this study we assessed, in the comb lesions of this experimental avian model, SDF-1/CXCR4, VEGFA/VEGF receptors, ET-1/ET receptors and PDGF-BB/PDGFR axes, to evaluate their potential involvement. We found that UCD-200 chickens display the same angiogenic impairment, associated with up-regulation of the pro-fibrotic molecules, mirroring the results of SSc patients. On these bases, since the very early stages of SSc and its pre-symptomatic period are still largely unknown, generally evading medical assessment, the UCD-200 chickens might represent an appropriate translational model, to investigate the early events of avian disease, and possibly identify potential targetable biomarkers to translate to human SSc.

\section{Material and methods}

\section{Patients and skin biopsies}

After approval of San Salvatore University Hospital ethics committee and written informed consent from patients, full-thickness skin biopsies were obtained from the involved forearm skin (skin score $1 / 2$ at the biopsy site) of 10 SSc patients with the diffuse cutaneous form, of recent onset (disease duration less than 3 years calculated since the first non-Raynaud's symptom of SSc) $[7,8]$. Demographic and clinical characteristics of the patients are shown in Table I.

Table I. Clinical and demographic features of the 10 early diffuse SSc patients

\begin{tabular}{|lcccccc|}
\hline $\begin{array}{l}\text { Gender/age } \\
\text { [years] }\end{array}$ & $\begin{array}{c}\text { Year of SSc } \\
\text { onset/disease } \\
\text { duration at skin } \\
\text { biopsy [years] }\end{array}$ & $\begin{array}{c}\text { MRSS/ } \\
\text { score at } \\
\text { skin biopsy }\end{array}$ & Autoantibodies & $\begin{array}{c}\text { Lung } \\
\text { involvement } \\
\text { HRCT/PFT }\end{array}$ & $\begin{array}{c}\text { Heart } \\
\text { involvement/ } \\
\text { scleroderma } \\
\text { renal crisis }\end{array}$ & $\begin{array}{c}\text { Raynaud's } \\
\text { phenomenon/ } \\
\text { digital ulcers }\end{array}$ \\
\hline F/46 & $2010 / 2$ & $12 / 2$ & ANA/Scl-70 & Normal/normal & Normal/no & Yes/no \\
\hline F/21 & $2009 / 3$ & $13 / 1$ & ANA/Scl-70 & Normal/normal & Normal/no & Yes/yes \\
\hline F/31 & $2011 / 1$ & $13 / 2$ & ANA/Scl-70 & Normal/normal & Normal/no & Yes/yes \\
\hline F/36 & $2010 / 2$ & $11 / 2$ & ANA/Scl-70 & Normal/normal & PAH/no & Yes/yes \\
\hline M/20 & $2010 / 2$ & $11 / 1$ & ANA/Scl-70 & Normal/normal & Normal/no & Yes/no \\
\hline F/41 & $2010 / 2$ & $15 / 2$ & ANA/Scl-70 & Normal/normal & Normal/no & No/no \\
\hline F/30 & $2010 / 2$ & $10 / 1$ & ANA/Scl-70 & Normal/normal & Normal/no & Yes/no \\
\hline F/21 & $2010 / 2$ & $09 / 1$ & ANA/Scl-70 & Normal/normal & Normal/no & Yes/no \\
\hline F/31 & $2009 / 3$ & $14 / 1$ & ANA/Scl-70 & Normal/normal & Normal/no & Yes/no \\
\hline F/42 & $2009 / 3$ & $16 / 2$ & ANA/Scl-70 & Fibrosis/normal & Normal/no & Yes/no \\
\hline
\end{tabular}

MRSS - modified Rodnan skin thickness score (maximum possible score 51), HRCT - high-resolution computed tomography, PFT pulmonary function test, ANA - antinuclear antibodies, ACA - anti-centromere antibodies, SCl-70 - anti-topoisomerase, PAH - pulmonary arterial hypertension. 
Due to the short half-life of the drugs used, patients discontinued oral vasodilators and intravenous prostanoids at least 1 week before biopsies. None took corticosteroids or immunosuppressive agents.

Skin samples from the same forearm region of age- and sex-matched healthy donors who underwent surgical treatment for trauma were obtained. For IF, the specimens were fixed in 10\% buffered formalin, dehydrated in graded alcohol series, and embedded in paraffin.

\section{Animals}

UCD-200 chickens and the healthy control chickens H.B15 were bred and maintained at the Central Laboratory Animal Facilities of Innsbruck Medical University. Breeding, housing, and collection of tissue samples were in accordance with the Animal Experiment Directive of the European Union (Directive 2010/63 EU) and the Austrian law on animals used for experimental purposes (Tierversuchsgesetz, BGBL I Nr. 501/1989 idF 2005 and BGBL I Nr. 114/2012).

Animals were sacrificed by cardiac exsanguination under Narkodorm anesthesia (Pentobarbital, CP-Pharma, Germany, 20 mg/kg i.v.). Combs were taken from 10 UCD-200 chickens with erythema of the comb, 10 with edema, and 10 with beginning necrosis at 1-2 weeks of age. Combs were also taken from 10 age-matched H.B15 healthy control chickens ( $\mathrm{cHC}$ ). All tissue specimens were fixed in neutral buffered formalin for $24 \mathrm{~h}$ and then embedded in paraffin.

\section{Immunofluorescence}

The immunofluorescence analysis was performed on paraffin sections (thickness $3 \mu \mathrm{m}$ ) using anti-von Willebrand factor (VWF), smooth-muscle actin- $\alpha$ ( $\alpha$-SMA), S100A4, SDF-1, CXCR4, VEGFA165, VEGFR2, VEGFR1, ET-1, ETAR, ETBR, PDGF$\mathrm{BB}$ and PDGFR antibodies. Chicken cross-reactive polyclonal antibodies directed against human vWF (1 : 50, Dako, Denmark), $\alpha$-SMA (1: 20, Abcam, UK), S100A4 (1: 20, Dako, Denmark), SDF-1 (1 : 50, Abcam, UK), CXCR4 (1: 20, LSBio, USA), VEGFA ( $1: 20$, SIGMA, USA), VEGFR-1 and 2 ( $1: 50$, Santa Cruz, USA), ET-1 (1: 50, SIGMA, USA), ETAR (1: 50, Gene Tex, Germany), ETBR (1: 20, Labome, USA), PDGF-BB ( $1: 50$, Bioss, USA) and PDGFR ( $1: 50$, Thermo Scientific, USA) were used due to the lack of specific anti-chicken antibodies.

Samples were successively stained with secondary antibodies (Alexa Fluor 488-conjugated and Alexa Fluor 555-conjugated, Invitrogen, USA) and counterstained using 4,6-diamidino-2-phenylindole (DAPI).

All antibodies and conjugates were diluted to predetermined optimal concentrations in phos- phate buffered saline (PBS, pH 7.2)/1\% IgG-free bovine serum albumin (BSA, Jackson ImmunoResearch Laboratories, Inc., USA).

Images were acquired using an Olympus BX53 fluorescence microscope.

\section{Enzyme-linked immunosorbent assay}

The concentrations of VEGFA, PDGF-BB, ET-1 and SDF-1 released in plasma of SSc patients, $\mathrm{hHC}$, UCD 200 and $\mathrm{CHC}$ were determined by enzyme-linked immunosorbent assay (ELISA), using antibodies showing chicken reactivity, according to the manufacturer's protocol (Mybiosource, California, USA). Individual measurements were done in triplicate and results were expressed as the median.

\section{Statistical analysis}

GraphPad Prism 5.0 software was used for statistical analyses. Results are expressed as median (range). Due to the non-parametric distribution of our data, the Mann-Whitney $U$ test was used as appropriate for analyses. Statistical significance was expressed by a $p$-value $<0.05$.

\section{Results}

\section{Perivascular fibroblasts in UCD-200 comb biopsies}

Using $v W F / \alpha-S M A$ double staining, which allows one to identify ECs and Ps respectively, we observed, in hHC skin (Figure $1 \mathrm{~A}$ ) and in $\mathrm{cHC}$ combs (Figure $1 \mathrm{~F}$ ), normally represented vessels, uniformly distributed in the intradermic area. By vWF/S100A4 double staining we noted that perivascular fibroblasts were normally present in the healthy skin of humans (Figure $1 \mathrm{~B}$ ) and in the combs of avian controls (Figure $1 \mathrm{G}$ ).

In SSc skin biopsies, we observed higher fluorescence intensity of S100A4 expression compared with hHC skin (Figures $1 \mathrm{~B}$ and D). Quantitative analysis, performed using ImageJ software, showed that S100A4 staining was significantly higher in SSc skin compared with $\mathrm{hHC}\left({ }^{* * *} p<\right.$ 0.0001) (Figure $1 \mathrm{E}$ ).

The biopsies of combs derived from UCD-200 with erythema (Figure $1 \mathrm{H}$ ) did not show any difference in either density or vessel morphology compared with those of $\mathrm{cHC}$ samples (Figure $1 \mathrm{~F}$ ); in contrast, in edematous combs, the vessel appeared dilated (Figure $1 \mathrm{~J}$ ) and, finally, in necrotic combs, marked rarefaction of vessels was observed (Figure 1 L). As regards perivascular fibroblasts, the S100A4 staining showed higher fluorescence intensity in all UCD-200 samples compared with $\mathrm{CHC}$ combs (Figures $1 \mathrm{G}, \mathrm{I}, \mathrm{K}, \mathrm{M}$ ). Quantitative analysis showed that S100A4 staining significantly increased in parallel with the se- 

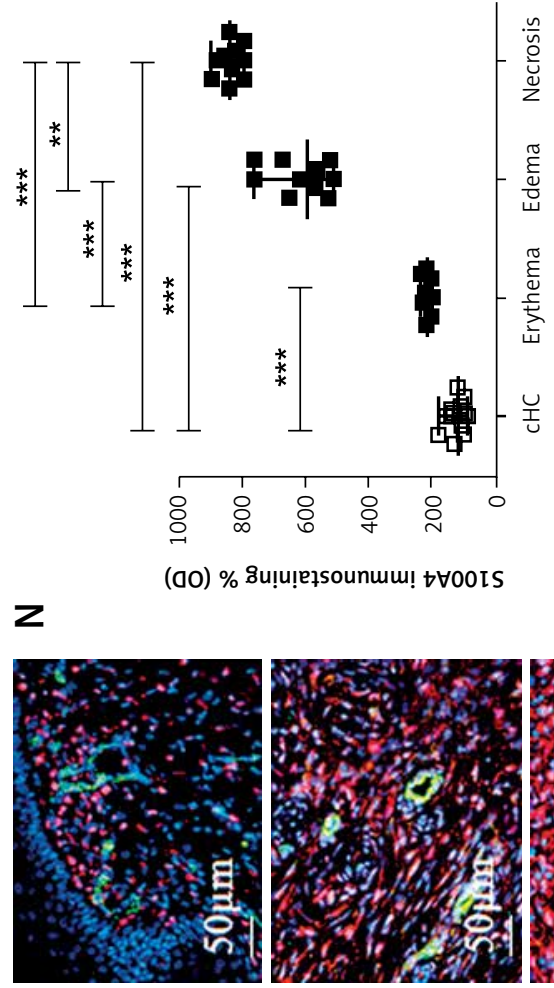

$\checkmark$

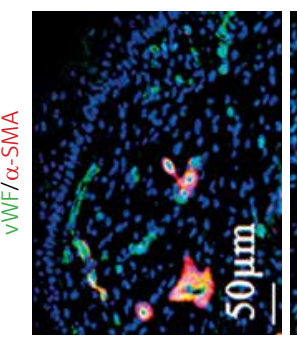

$\leftarrow$

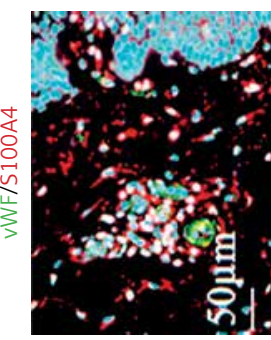

$\infty$

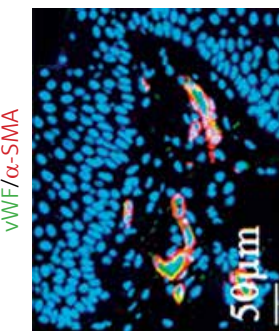

$\varangle$

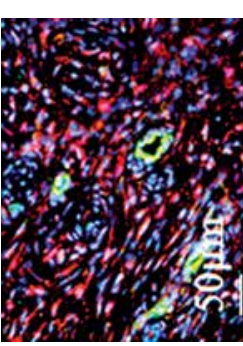

$-$

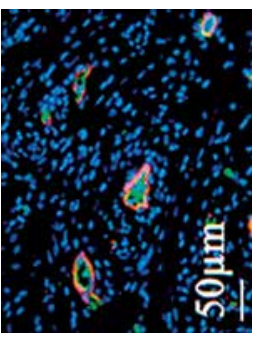

I

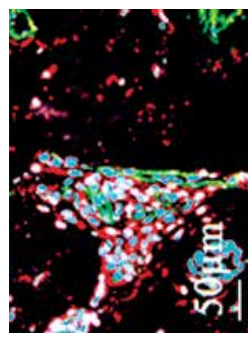

○

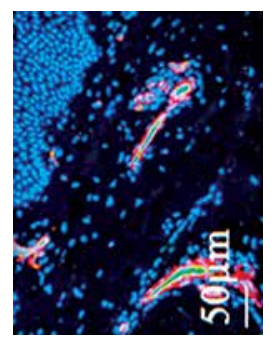

$\checkmark$

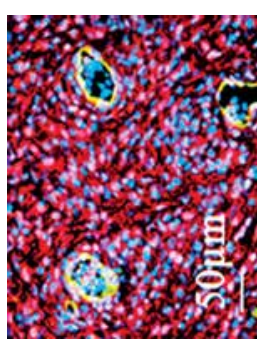

$\checkmark$

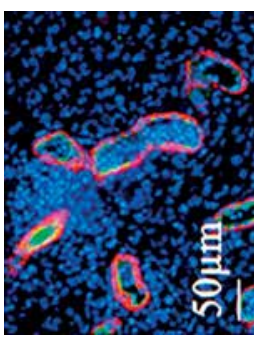

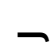

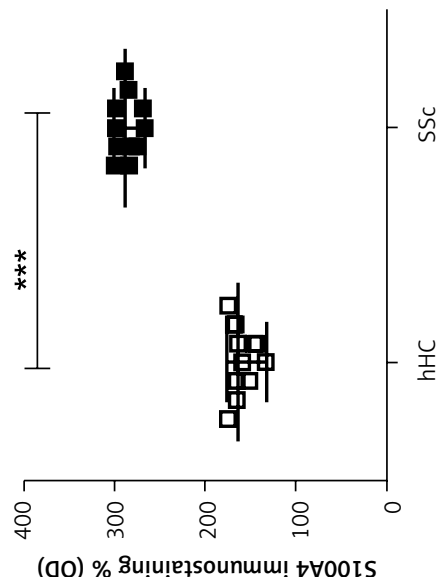

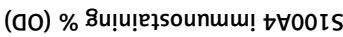
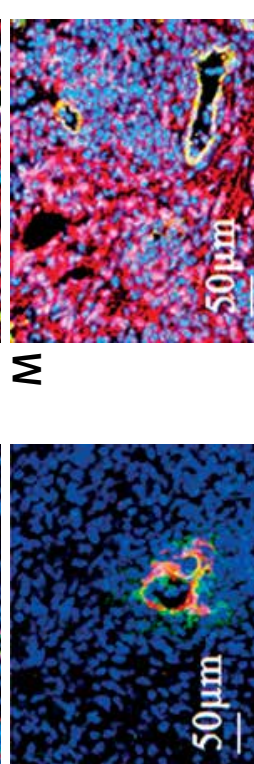

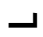

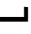

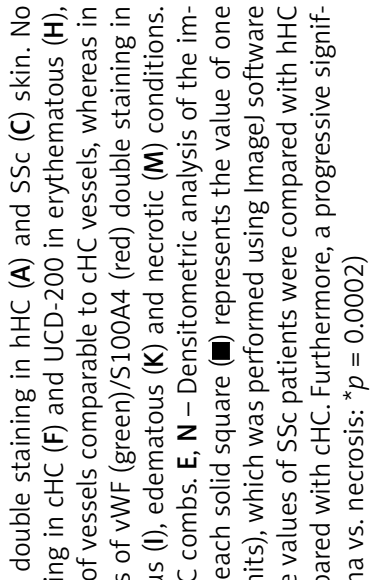

:

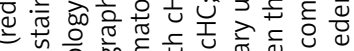

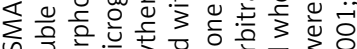

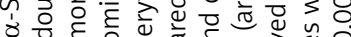

당.

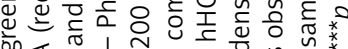

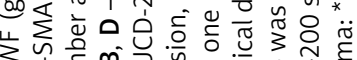

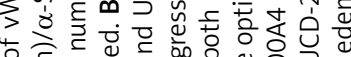

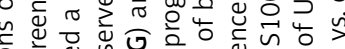

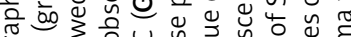

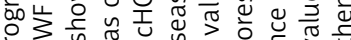

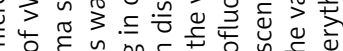

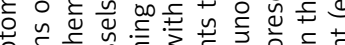

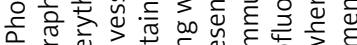

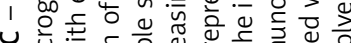

《ं

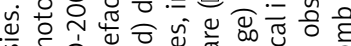

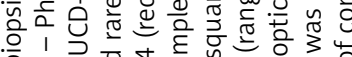

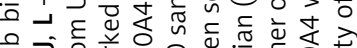

है

証变

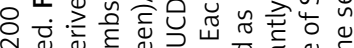

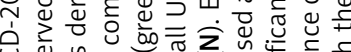

ป气

ऽ

ษ。

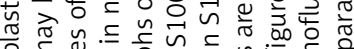

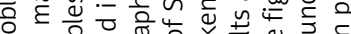

흔

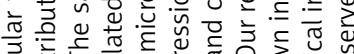

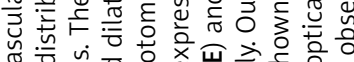

婂

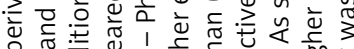

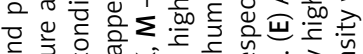

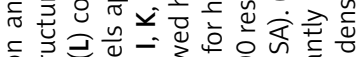

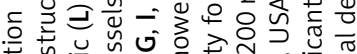

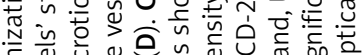

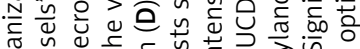

के एँ

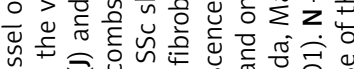

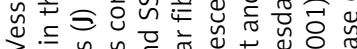

ن.

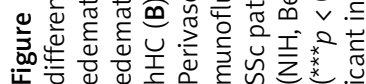


verity of the disease $\left({ }^{* *} p=0.0002 ;{ }^{* * *} p<0.0001\right)$ (Figure $1 \mathrm{~N}$ ).

\section{Angiogenic molecules in UCD-200 combs}

The SDF-1 and CXCR4 expression in SSc skin (Figures 2 D, E, F) was significantly higher than the expression in hHC skin (Figures $2 \mathrm{~A}, \mathrm{~B}, \mathrm{C}$ ). Quantitative analysis performed using ImageJ software showed that in SSc skin the expression of both SDF-1 and CXCR4 was significantly higher than in hHC skin $\left.{ }^{* * *} p<0.0001\right)$ (Figures $2 \mathrm{G}, \mathrm{H}$ ).

In the $\mathrm{CHC}$ combs, weak constitutive expression of SDF-1 (Figure $2 \mathrm{I}$ ) and CXCR4 (Figure $2 \mathrm{~J}$ ) was observed in ECs and pericytes of dermal vessels and in fibroblasts.

In combs from UCD-200 chickens, at the erythematous stage of disease, the number of SDF$1+/$ CXCR4+ cells (Figures $2 \mathrm{~L}, \mathrm{M}, \mathrm{N}$ ) was similar to that of healthy controls (Figures $2 \mathrm{I}, \mathrm{J}, \mathrm{K}$ ). The number of total SDF-1+/CXCR4+ cells was increased in the combs of chickens with edema (Figures 2 $\mathrm{O}, \mathrm{P}, \mathrm{Q}$ ) and at the ischemic stage (Figures $2 \mathrm{R}, \mathrm{S}$, T). Quantitative analysis in UCD-200 showed that the SDF-1 and CXCR4 expression significantly increased in parallel with the severity of the disease ${ }^{* *} p=0.0002 ;{ }^{* * *} p<0.0001$ ) (Figures $2 \mathrm{U}, \mathrm{V}$ ).

As regards VEGFA and VEGFR2/VEGFR1 expression, these molecules were weakly constitutively expressed in hHC skin (Figures 3 A1-6). Both VEGFA and VEGFR expression was strongly increased in the SSc skin (Figures 3 B1-6), and the quantitative analysis confirmed that, in SSc skin, the expression of the VEGFA/VEGFR2/VEGFR1 axis was significantly higher than $\mathrm{hHC}$ skin $\left({ }^{* * *} p<\right.$ 0.0001) (Figures 3 C, D, E).

The same molecules were constitutively expressed in $\mathrm{CHC}$ combs (Figures $3 \mathrm{F1}-6$ ). Both VEGFA and VEGFR expression was strongly increased in the edematous and in the necrotic combs of UCD200 (Figures $3 \mathrm{H} 1-6$ and Figures 3 I1-6). Mirroring the results obtained with SDF-1/CXCR4 staining, quantitative analysis showed that, in UCD-200, the increase of the VEGFA/VEGFR2/VEGFR1 axis correlated with the severity of lesions ${ }^{* *} p=$ $0.0002 ;{ }^{* * *} p<0.0001$ ) (Figures $3 \mathrm{~J}, \mathrm{~K}, \mathrm{~L}$ ).

\section{ET-1/ETAR/ETBR assessment in UCD-200 combs}

The ET-1, ETAR and ETBR staining showed a weak constitutive expression in $\mathrm{hHC}$ skin (Figures 4 A1-6). In SSc skin (Figures 4 B1-6), the ET-1 and ETR expression was significantly higher than in hHC-skin. Quantitative analysis showed that, in SSc skin, the ET-1/ETAR/ETBR axis was significantly higher than in $\mathrm{hHC}$ skin ${ }^{* * *} p<0.0001$ ) (Figures $4 \mathrm{C}, \mathrm{D}, \mathrm{E})$.

In $\mathrm{CHC}$ combs (Figures 4 F1-6), ET-1, ETAR and ETBR were constitutively expressed. In the erythem- atous phases of the avian disease, we found an increase of both ET-1 and ETR expression in vascular cells and in perivascular fibroblasts of UCD-200 (Figures $4 \mathrm{G} 1-6$ ). In both the edematous (Figures $4 \mathrm{H1}-6$ ) and necrotic phase (Figures 4 I1-6), the number of ET-1+ and ETR+ cells increased progressively. Quantitative analysis showed that, in UCD-200, the increase of the ET-1/ETAR/ETBR axis correlated with the severity of lesions $\left({ }^{*} p=0.0004\right.$; ${ }^{* *} p=0.0002 ;{ }^{* * *} p<0.0001$ ) (Figures $4 \mathrm{~J}, \mathrm{~K}, \mathrm{~L}$ ).

\section{PDGF-BB/PDGFR assessment in UCD-200 combs}

In the hHC skin, constitutive weak expression of both PDGF-BB and PDGFR was observed (Figures $5 \mathrm{~A}-\mathrm{C}$ ). Of note, in SSc skin (Figures $5 \mathrm{D}-\mathrm{F}$ ), we observed significantly higher PDGF-BB and PDGFR expression than in hHC skin. Quantitative analysis mirrored the results obtained with SDF-1/ CXCR4 staining (Figures $5 \mathrm{G}, \mathrm{H}$ ).

The PDGF-BB/PDGFR double staining, in $\mathrm{CHC}$ comb biopsies, showed that PDGF-BB was constitutively expressed in ECs of vessels and PDGFR was expressed in Ps and fibroblasts (Figures 5 I-K). In UCD-200 combs characterized by erythema, both PDGF-BB and PDGFR expression was comparable with $\mathrm{CHC}$ expression (Figures $5 \mathrm{~L}-\mathrm{N}$ ). We observed a strong increase of both PDGF-BB and PDGFR expression in UCD-200 combs characterized by edema (Figures $5 \mathrm{O}-\mathrm{Q}$ ) when compared with healthy chickens, and the increase of expression was significantly higher in necrotic combs when compared with the other phases of disease (Figures $5 \mathrm{R}-\mathrm{T}$ ). Quantitative analysis revealed that, in UCD-200, the PDGF-BB/PDGFR axis significantly increased with the severity of the lesions ${ }^{* *} p=0.0002 ;{ }^{* * *} p<0.0001$ ) (Figures $\left.5 \mathrm{U}, \mathrm{V}\right)$.

\section{SDF-1, VEGFA, ET-1 and PDGF-BB plasma concentration in UCD-200}

Using human and chicken ELISA kits, we evaluated plasma concentrations of VEGFA, SDF-1, PDGF-BB and ET-1.

There was no statistically significant difference in SDF-1 plasma concentration among the investigated groups [Human SDF-1 ng/ml: 2.4 (2.2-2.5) in hHC; $3.0(2.3-3.8)$ in SSc; chicken SDF-1 ng/ $\mathrm{ml}$ : $3.5(1.4-6.3)$ in $\mathrm{CHC} ; 4.25$ (1.5-6.3) in UCD200 with erythema; 3.3 (1.5-6.3) in UCD-200 with edema; 1.7 (0.9-6.3) in UCD-200 with necrosis, $p=\mathrm{NS}$ ] (Figures $6 \mathrm{~A}, \mathrm{~B}$ ). The VEGFA (Figure $6 \mathrm{C}$ ) and PDGF-BB (Figure $6 \mathrm{G}$ ) levels in SSc plasma were significantly higher than in hHC [Human VEGFA ng/ml: 111.5 (100.0-132.0) in hHC vs. 417.00 (403.0453.0) in SSc; $p<0.0001$. Human PDGF-BB pg/ml: $2163(2013-2433)$ in hHC vs. 2826 (2763-2877) in SSc; $p=0.0001]$. As regards chicken plasma val- 


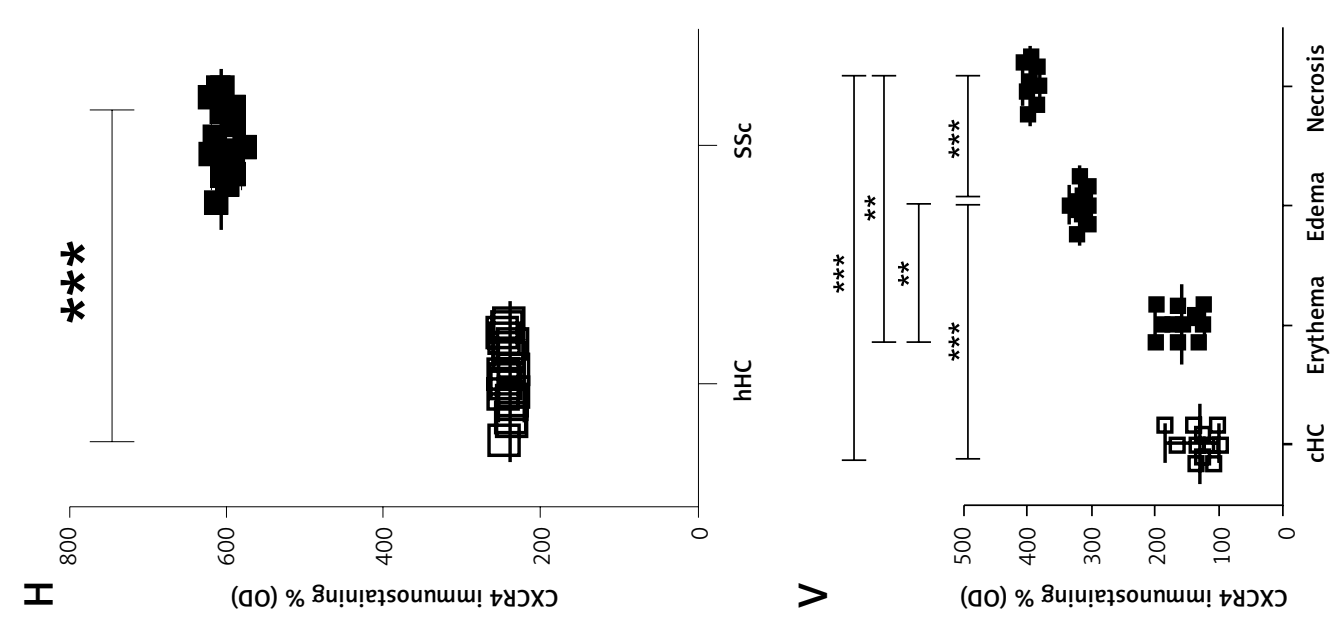

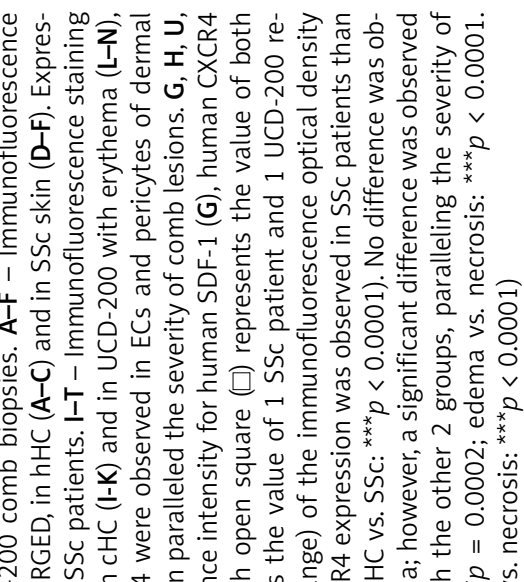

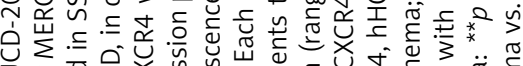

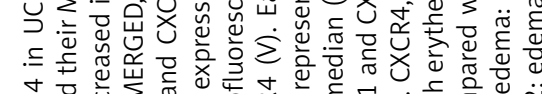
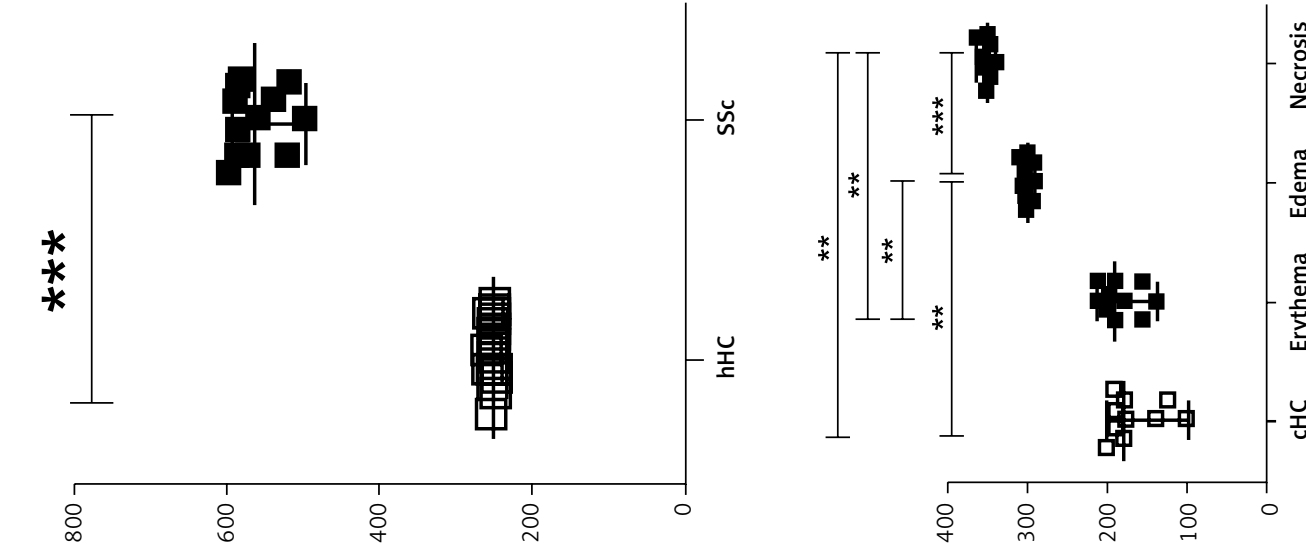

$\circlearrowleft$

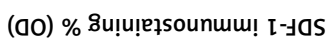

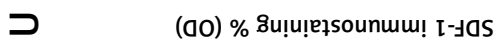

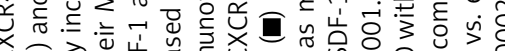

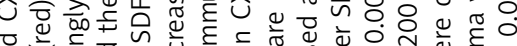

¿

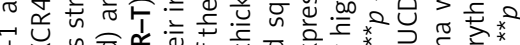

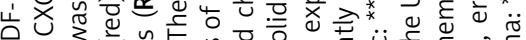

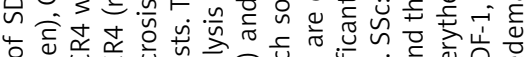

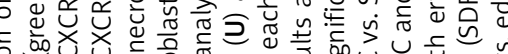

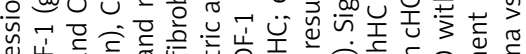

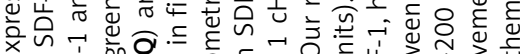

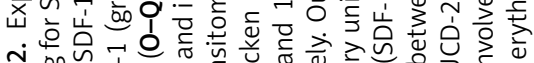

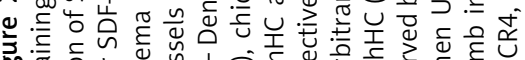

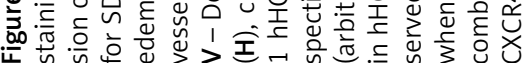
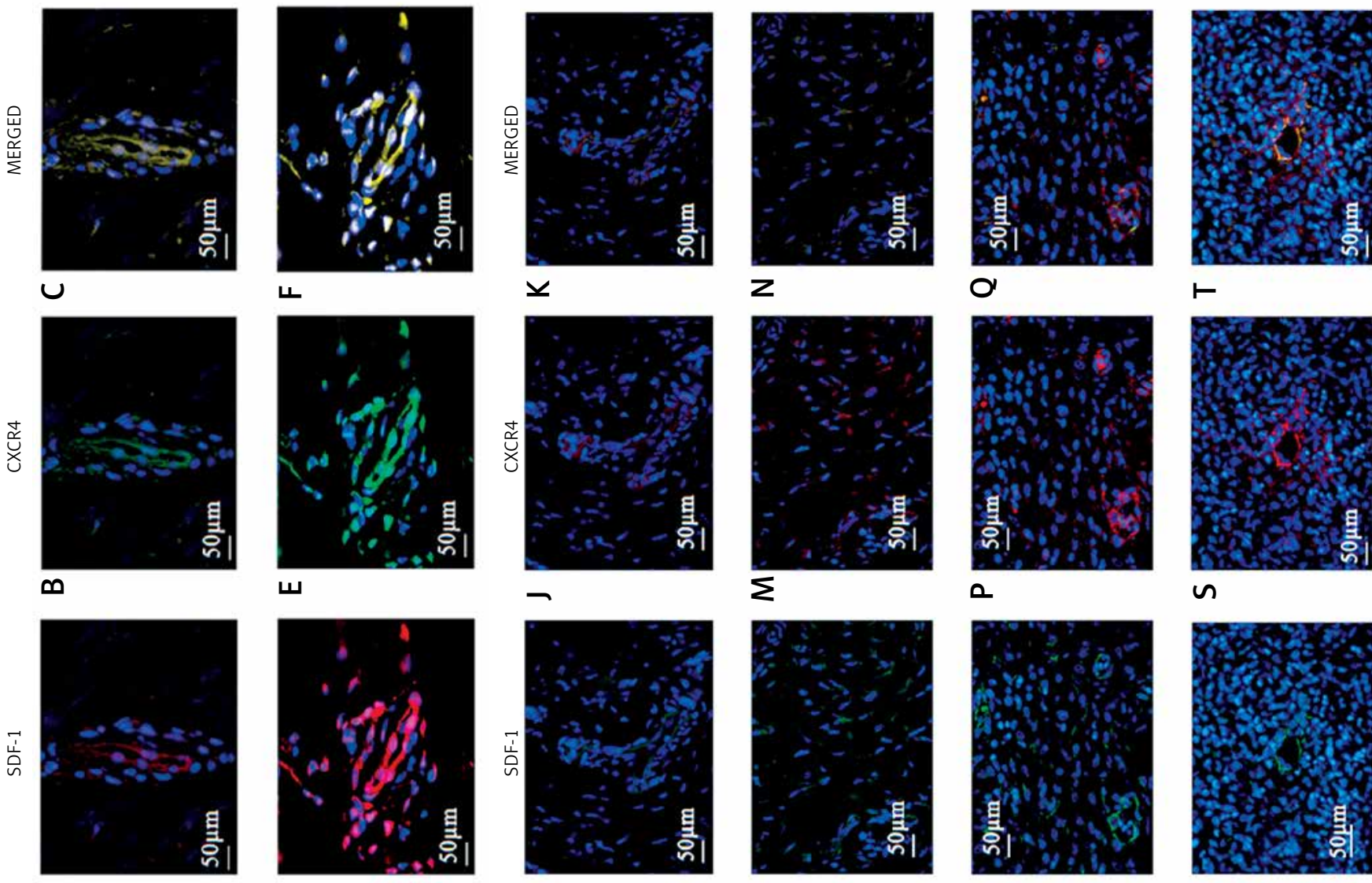

口
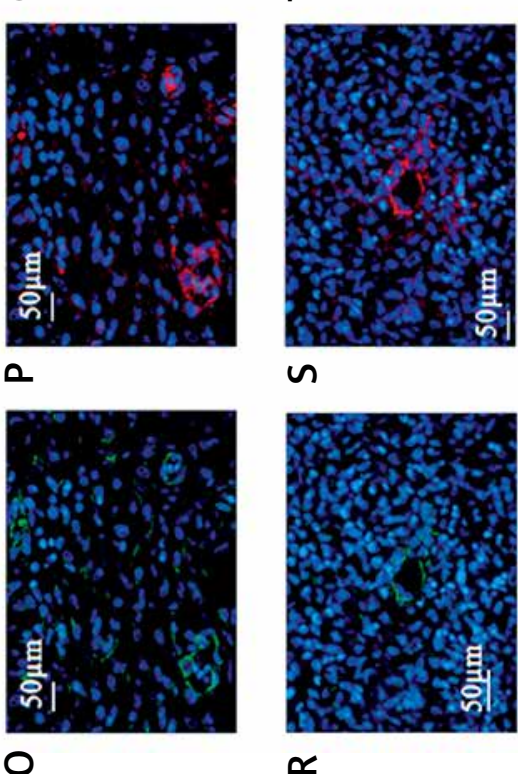

$\propto$ 
ues, higher concentrations of VEGFA (Figure $6 \mathrm{D}$ ) and PDGF-BB (Figure $6 \mathrm{H}$ ) were found only in UCD-200 samples affected by edematous and necrotic stages, when compared with both the control chickens and the chickens with the erythematous stage [chicken VEGFA ng/ml: 28.5 (24.0-38.0) in $\mathrm{CHC} ; 35.5$ (24.0-45.0) in UCD-200 with erythema; 144.0 (125.0-175.0) in UCD-200 with edema; 197.5 (185.0-254.0) in UCD-200 with necrosis]; [chicken PDGF-BB pg/ml: 445.5 (324.0-600.0) in CHC; 589.0 (389.0-654.0) in UCD-200 with erythema; 1588.0 (1187.0-1923.0) in UCD-200 with edema; 2978.0 (2634.0-3765.0) in UCD-200 with necrosis]. The ET-1 (Figure 6 E) plasma concentration in SSc patients was significantly higher than in hHC [SSc ET-1 pg/ml: 1.1 (1.0-1.3) in hHC vs. 2.6 (2.2-2.8) in SSc; $p<0.0001]$. ET-1 chicken plasma concentration (Figure $6 \mathrm{E}$ ) was significantly elevated in all UCD-200 samples, increasing with disease progression [chicken ET-1 pg/ml: 0.7 (0.5-0.9) in CHC; 1.8 (1.6-2.0) in UCD-200 with erythema; 4.1 (3.5-4.3) in UCD-200 with edema; 5.5 (5.0-6.1) in UCD-200 with necrosis] (Figure 6 F).

\section{Discussion}

Many efforts have been made, in the last years, by the scientific community to identify a good animal model to study the pathogenesis of SSc. Although many brilliant in vivo studies on different animal models have shown the ability of several drugs to temper the fibrotic process, until now, the translation of these data to the clinical setting, by interventional studies, has failed to demonstrate any consistent efficacy in ameliorating tissue fibrosis or modifying the evolution of human SSc [9].

This discrepancy raises the need to find an experimental model of the disease displaying all the hallmarks of human SSc, including organ manifestations [10, 11]. Nevertheless, given the awareness that SSc is more than a fibrotic condition, with a pivotal vascular involvement, animal models that recapitulate vasculopathy, inflammation and fibrotic reactions should be considered the 'reference points'.

In this setting, the UCD-200 chickens show microvascular damage with apoptosis of endothelial cells and microvascular occlusions, followed by prominent perivascular lymphocytic infiltration involving skin and viscera $[12,13]$. Early phases of the animal disease include erythema, edema, and finally necrosis of the comb, thus mirroring Raynaud's phenomenon in SSc. Furthermore, pronounced skin fibrosis and thickening of different tissues may be observed. About $90 \%$ of the birds are afflicted by fibrosis of internal organs (notably esophagus, lung, kidney) at the age of 5 weeks [4].

Taken together, this sequence of vasculopathy, inflammation, and subsequent fibrosis suggests that the UCD-200 chickens may be considered a good model of human scleroderma, despite the fact that the avian disease shows an accelerated course compared with the human SSc. Of note, the vasculopathic and inflammatory stages of the human disease are absent in other animal models [14]

It must be pointed out that this avian model shows some specific difficulties, such as the breeding process and chickens' size, so doing the research with UCD-200 chickens is more expensive than with mice. Furthermore, tools to study signaling in an avian background are limited. Taken together, these problems may explain why the molecular basis of avian SSc-like disease pathogenesis is still unknown.

To better understand if this avian model could be considered a good model of human scleroderma, independent of the reported issues, we analyzed in UCD-200 chickens the behavior of several molecules, which are strongly impaired in human SSc and linked to its pathogenesis, aimed at identifying possible similarities between avian and human SSc. The biopsy specimens were obtained sequentially, when the UCD-200 chickens first showed erythema, subsequently edema and then necrosis in the comb [15].

Our data showed that in the edematous phase, dermal vessels were dilated, and we observed a progressive loss of vessels until the necrotic phase. Of note, in edematous biopsies the number of perivascular fibroblasts was significantly higher than in $\mathrm{CHC}$, and the intensity of S100A4 increased progressively to reach the highest levels in the necrotic UCD-200 combs, when the avascular zone was present. Similarly, the SSc skin showed significantly higher S100A4 fluorescence intensity than hHC skin.

A key molecule in human SSc is SDF-1. It is a CXC chemokine that exerts its effect via the binding of CXCR4 [13], thus mediating the endothelial progenitor cells' mobilization and differentiation [16]. Interestingly, it was observed that SDF-1 acts locally within the hypoxic environment and stimulates direct differentiation of the recruited bone marrow cells into endothelial cells $[17,18]$. SDF-1 is strongly expressed in ECs [19] and Ps [20] of vessels, and it has been suggested that SDF-1 detected in ECS may result from the uptake of SDF-1 released by surrounding cells such as Ps [21].

We previously found that SDF-1 and CXCR4 were up-regulated in the skin of patients with early (edematous) SSc, both in the diffuse and limited cutaneous forms, and they progressively decreased with the duration of the disease [19]. Mirroring these results, we found that SDF-1 and CXCR4 were strongly expressed in the skin of patients with diffuse SSc. Of note, in UCD-200, we noted higher expression of both SDF-1 and CXCR4 in vascular cells, when the disease was limited to 

resemble the early phase of human systemic sclerosis

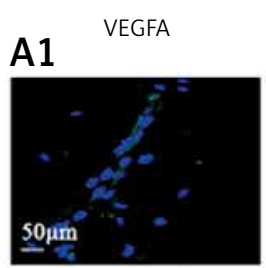

A2
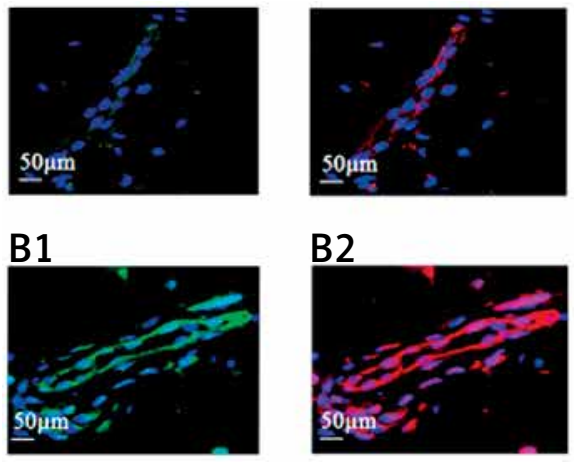

B2

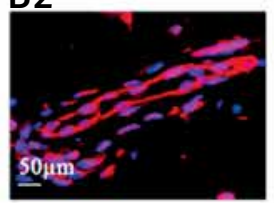

C

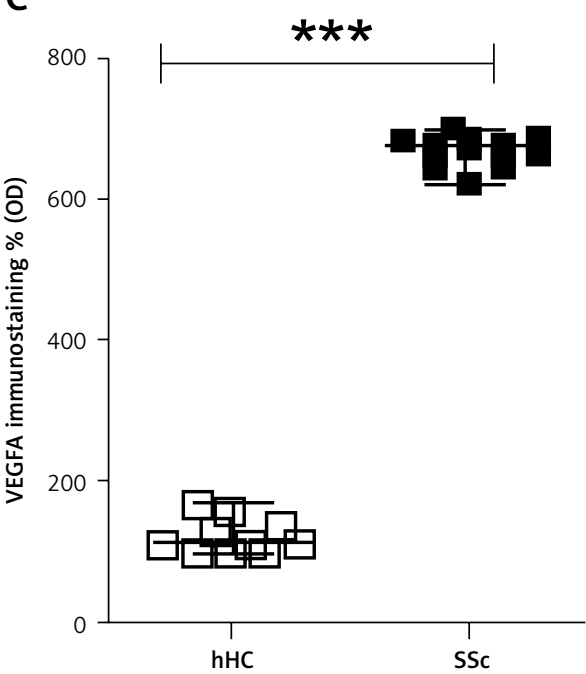

VEGFA

F1

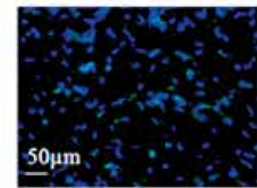

G1

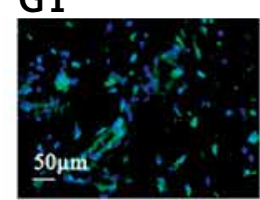

H1

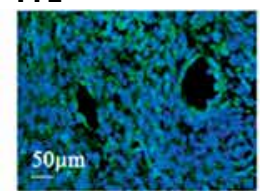

I1

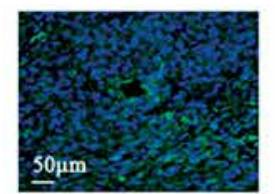

F2

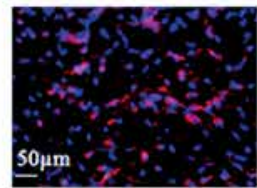

G2

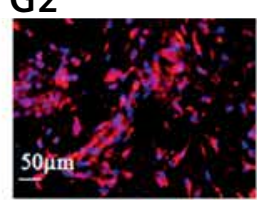

$\mathrm{H} 2$

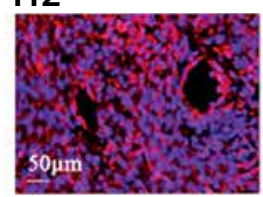

12

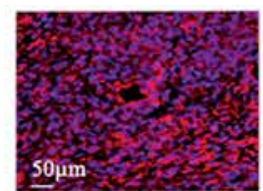

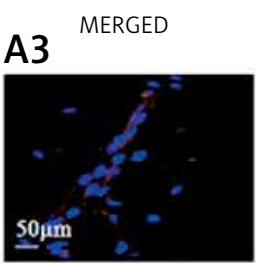

A4

VEGFA
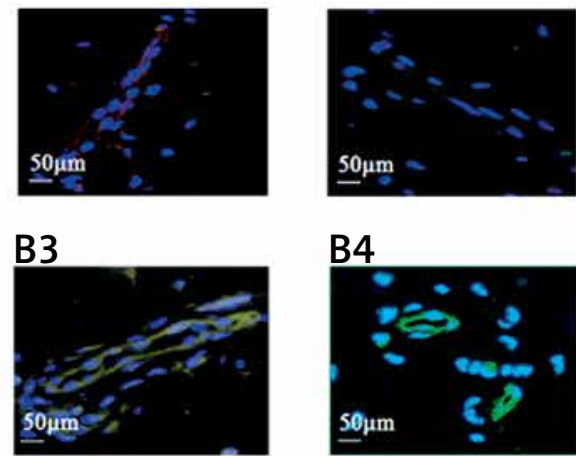

B4

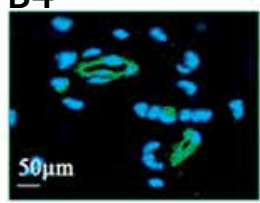

D
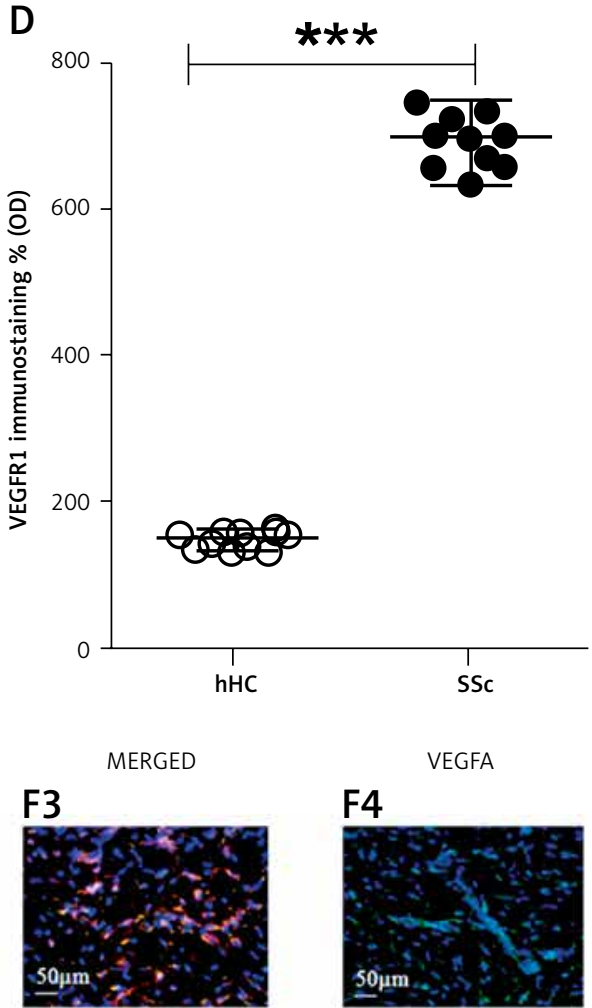

G3

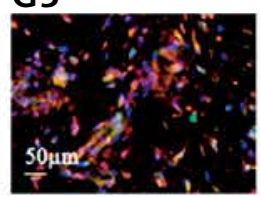

$\mathrm{H} 3$

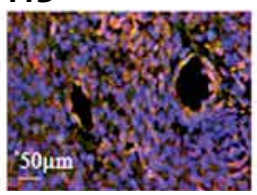

13

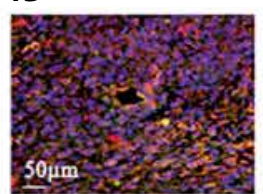

F4

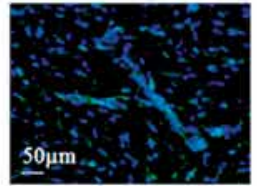

G4

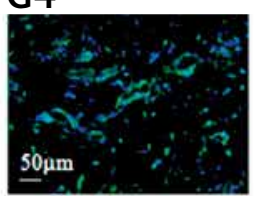

$\mathrm{H} 4$

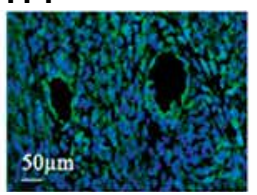

14

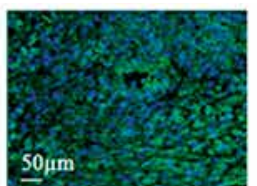

A5

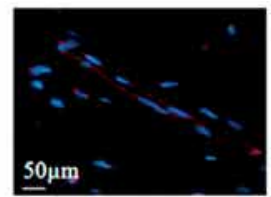

B5

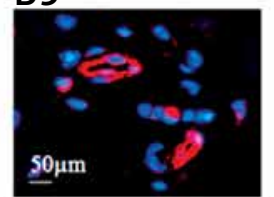

E

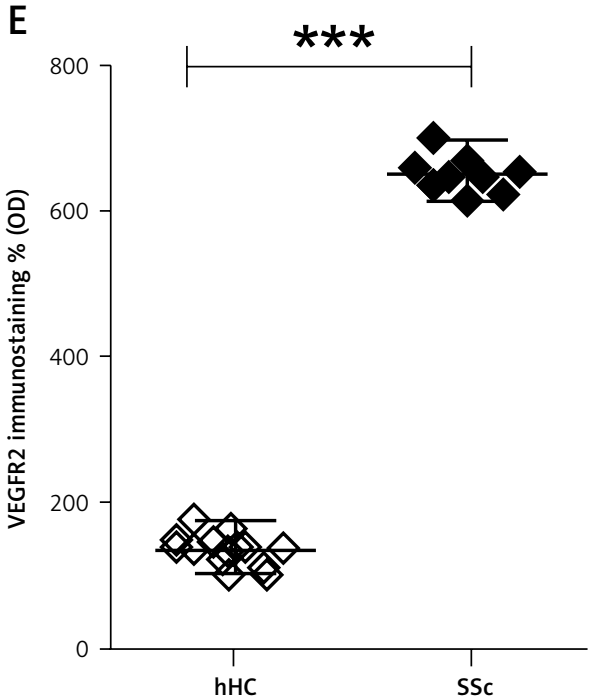

VEGFR2
F5

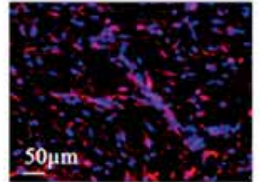

G5

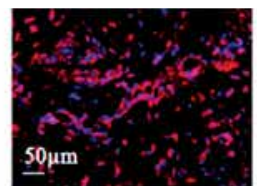

H5

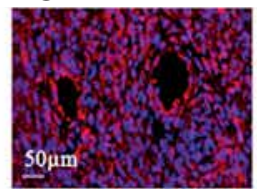

15

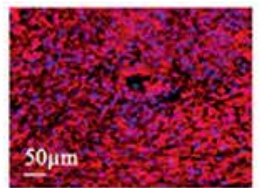

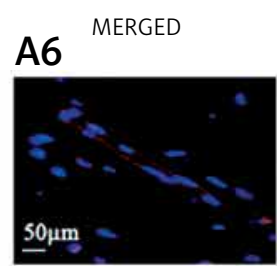

B6

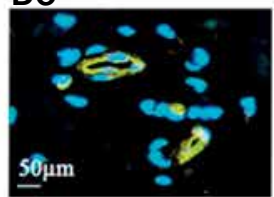

\section{sempris}

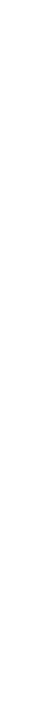

(


Figure 3. Cont.
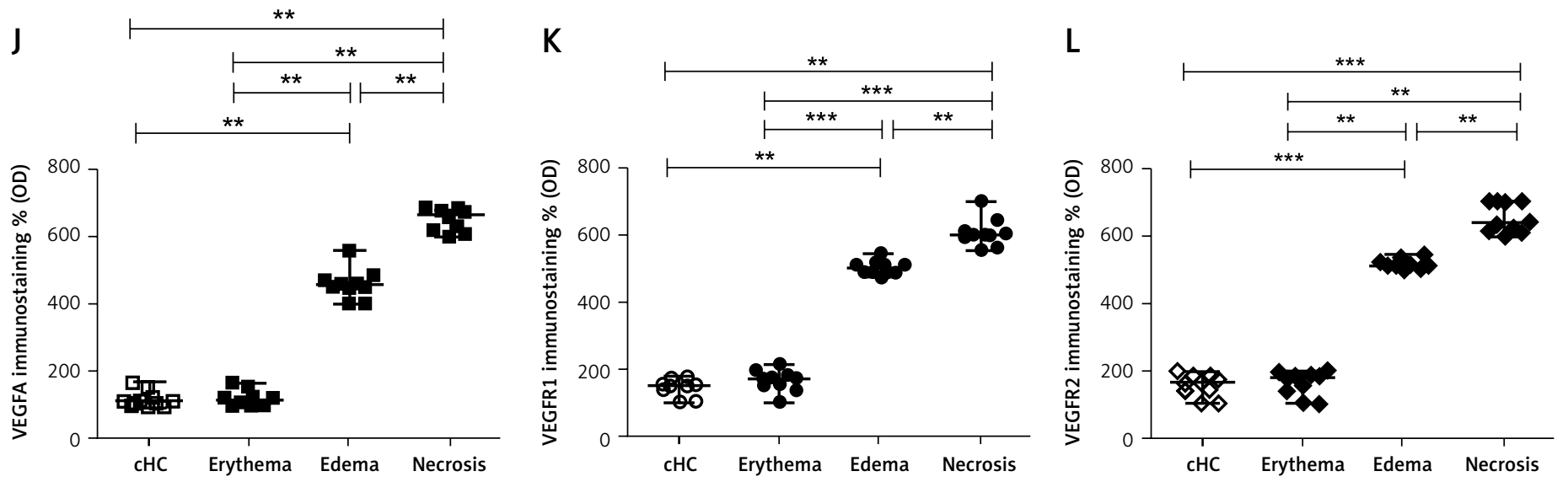

Figure 3. Expression of VEGFA, VEGFR1 and VEGFR2 in UCD-200 comb biopsies. A, B - This figure presents the staining of VEGFA (1), VEGFR1 (2), their MERGED (3) and the staining of VEGFA (4), VEGFR2 (5), their MERGED (6), in hHC (A) and in SSc (B) patients. Expression of both VEGFA and VEGFRs was considerably higher in SSc patients than in hHC. F, G, H, I - This figure presents the staining of VEGFA (1), VEGFR1 (2), their MERGED (3) and the staining of VEGFA (4), VEGFR2 (5), their MERGED (6), in CHC (F) and in UCD-200 combs with erythema $(\mathbf{G})$, edema $(\mathbf{H})$ and necrosis (I). The VEGFA and VEGFRs expression was considerably higher in both the edematous and the necrotic combs of UCD-200 than in both CHC and erythematous conditions. C, J - Densitometric analysis of the immunofluorescence intensity of human (C) and chicken (J) VEGFA. Each open square $(\square)$ represents the VEGFA value of both 1 hHC and $1 \mathrm{cHC}$; each solid square ( represents the VEGFA value of 1 SSc patient and 1 UCD-200, respectively. Our results are expressed as median (range) of the immunofluorescence optical density (arbitrary units). The expression of VEGFA in SSc patients was significantly higher than in hHC (VEGFA, hHC vs. SSc: ${ }^{* * *} p$ $<0.0001$ ). No difference was observed between CHC and UCD-200 erythematous samples. However, significantly higher VEGFA optical density was observed in erythematous UCD-200 samples than in both edematous and necrotic UCD-200 samples (VEGFA, erythema vs. edema: ${ }^{* *} p=0.0002$; edema vs. necrosis: ${ }^{* *} p=0.0002$ ). $\mathbf{D}$ and $\mathbf{K}-$ Densitometric analysis of the immunofluorescence intensity of human (D) and chicken (K) VEGFR1. Each open dot (O) represents the VEGFR1 value of both $1 \mathrm{hHC}$ and $1 \mathrm{cHC}$; each solid dot (๑) represents the VEGFR1 value of 1 SSc patient and 1 UCD-200 sample respectively. Our results are expressed as median (range) of the immunofluorescence optical density (arbitrary units). The VEGFR1 expression was significantly higher in SSc patients than in hHC (VEGFR1, hHC vs. SSc: ${ }^{* *} p<0.0001$ ). No difference was observed between $\mathrm{CHC}$ and UCD-200 erythematous samples. However, significantly higher VEGFR1 optical density was observed in the erythematous UCD-200 samples than in the edematous and necrotic UCD-200 samples (VEGFR1, erythema vs. edema: ${ }^{\star \star *} p<0.0001$; edema vs. necrosis: ${ }^{* *} p=0.0002$ ), paralleling the severity of comb involvement. $E, L$ - Densitometric analysis of immunofluorescence intensity of human (E) and chicken (L) VEGFR2. Each open rhombus $(\diamond)$ represents the VEGFR2 value of both $1 \mathrm{hHC}$ and $1 \mathrm{cHC}$; each solid rhombus ( $)$ represents the VEGFR2 value of 1 SSc patient and 1 UCD-200 sample respectively. Our results are expressed as median (range) of the immunofluorescence optical density (arbitrary units). The expression of VEGFR2 was significantly higher in SSc patients than in hHC (VEGFR2, hHC vs. SSc: $\left.{ }^{* * *} p<0.0001\right)$. No difference was observed between $C H C$ and UCD-200 erythematous samples. However, significantly higher VEGFR2 optical density was observed in the erythematous UCD-200 samples than in the edematous and the necrotic UCD-200 samples (VEGFR2, erythema vs. edema: ${ }^{* *} p=0.0002$; edema vs. necrosis: ${ }^{* *} p=0.0002$ ) 

resemble the early phase of human systemic sclerosis

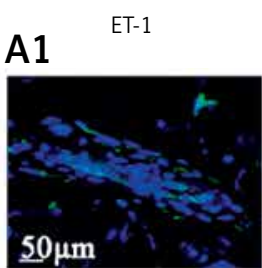

B1

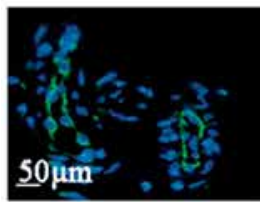

A2

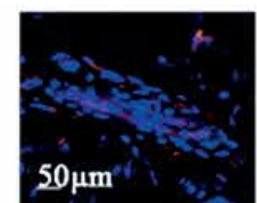

B2

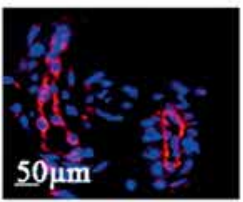

C

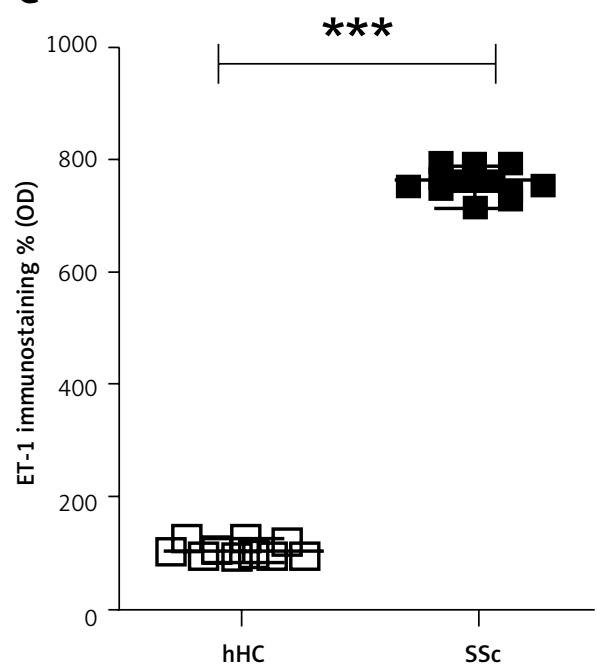

ET-1

F1

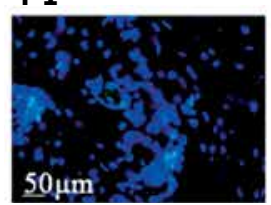

G1

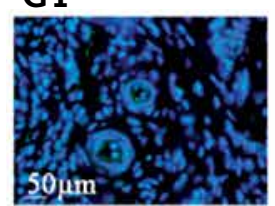

H1

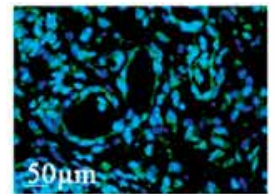

I1

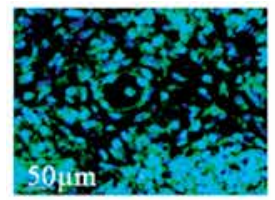

F2

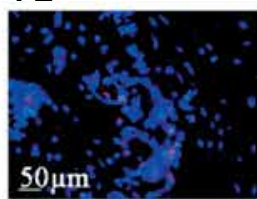

G2

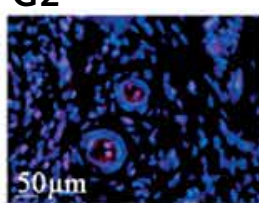

$\mathrm{H} 2$

12

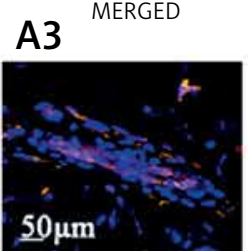

B3

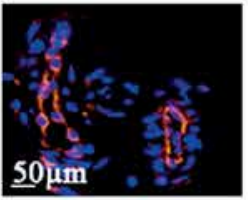

D

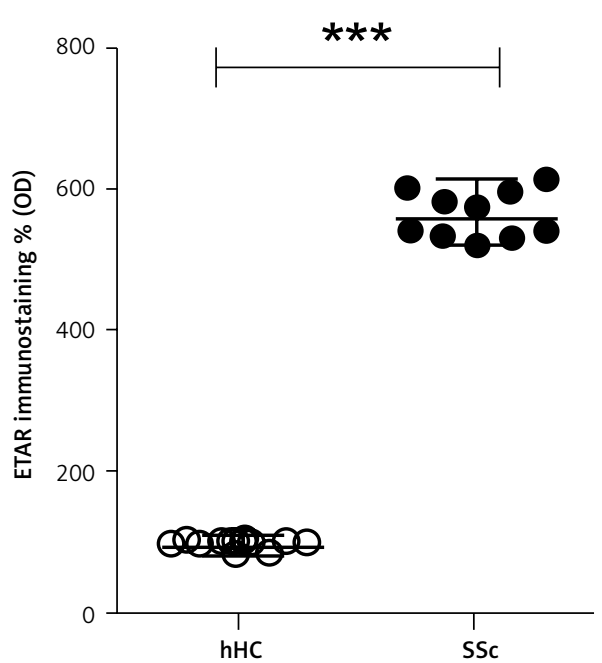

MERGED
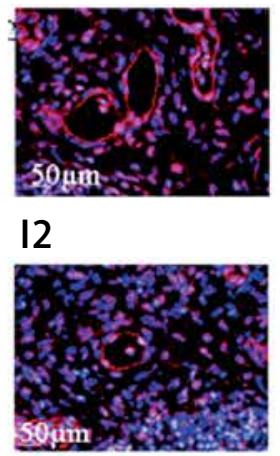

A4

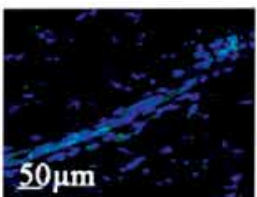

B4

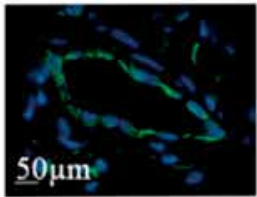

$\underline{50} \mu \mathrm{m}$
F3

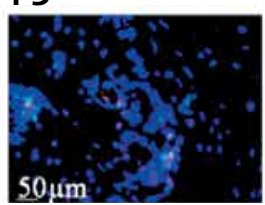

G3

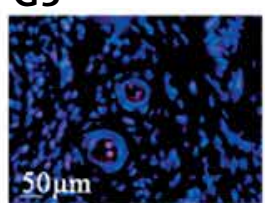

\section{H3}

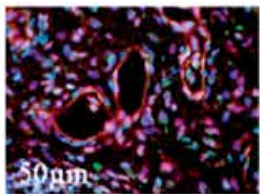

13

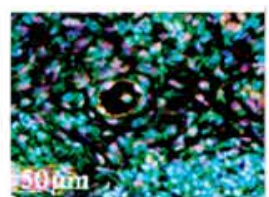

F4

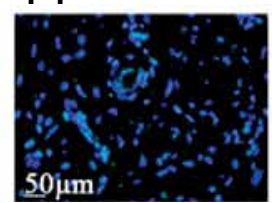

G4

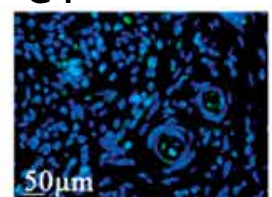

H4

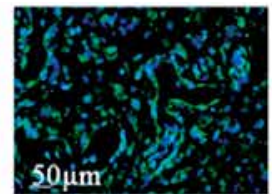

14

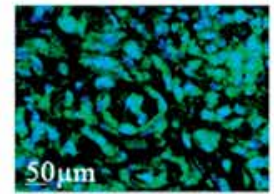

A5

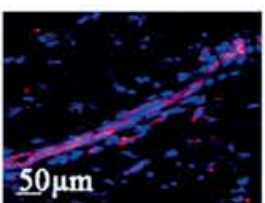

B5

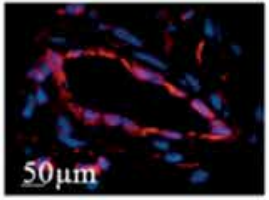

E

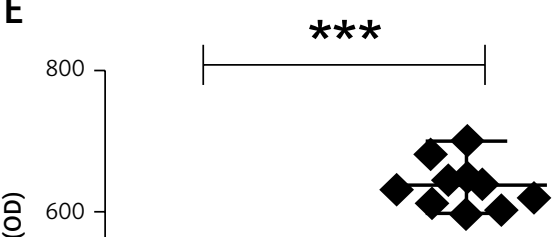

A6

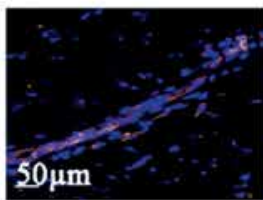

B6

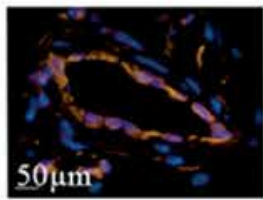

$500 \mathrm{~m}$

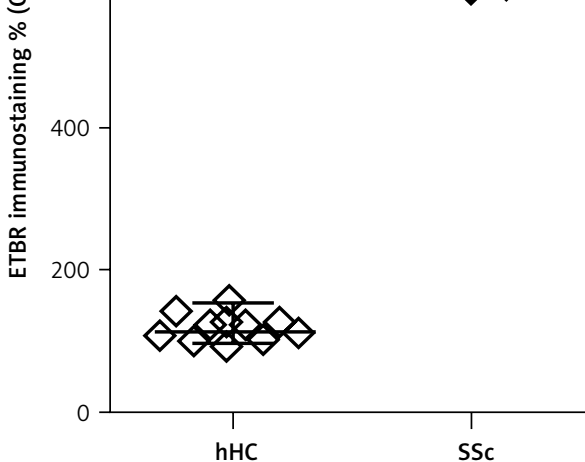

F5

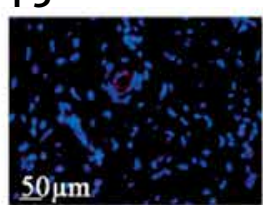

G5

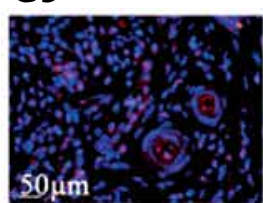

H5

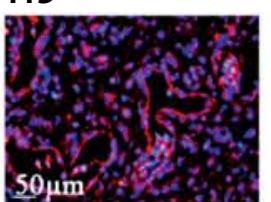

15

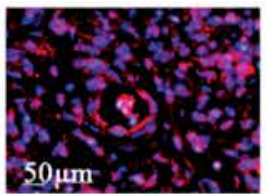

F6

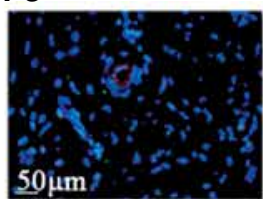

G6

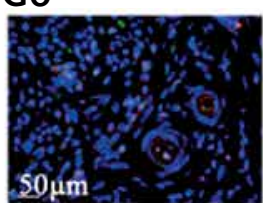

H6

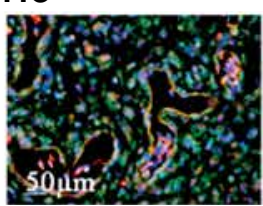

16

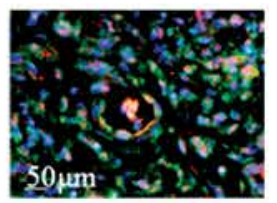


Figure 4. Cont.

J

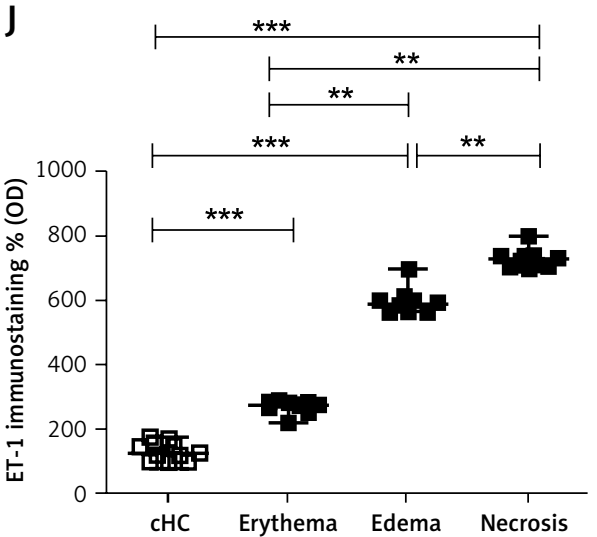

K

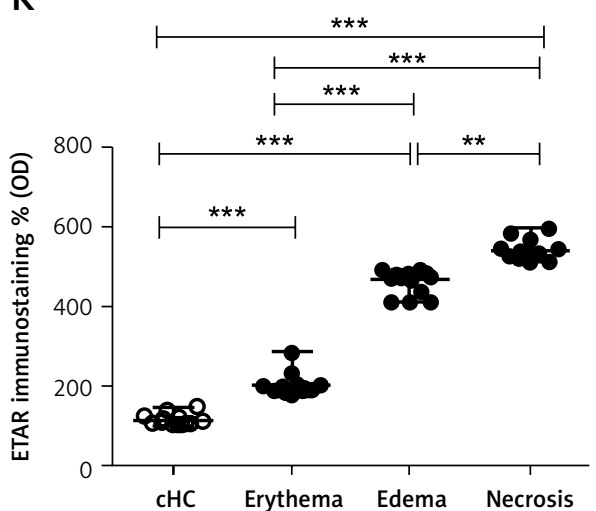

$\mathrm{L}$

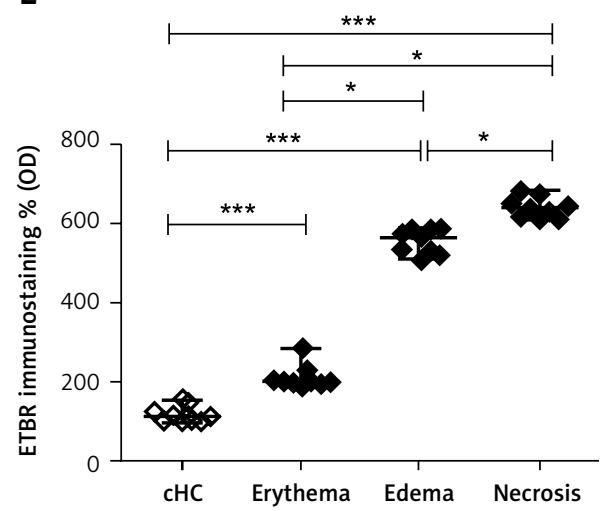

Figure 4. Expression of ET-1, ETAR and ETBR in UCD-200 comb biopsies. A, B - The figure shows the staining of ET-1 (1), ETAR (2) their MERGED (3) and of ET-1 (4), ETBR (5), their MERGED (6), in hHC (A) and in SSc patients (B). The expression of ET-1, ETAR and ETBR in SSc patients was significant higher than in hHC. F, G, H, I- The figure shows the staining of ET-1 (1), ETAR (2) their MERGED (3) and of ET-1 (4), ETBR (5), their MERGED (6), in cHC (F) and in UCD-200 combs with erythema (G), edema (H) and necrosis (I). The increased expression of the ET-1/ETR axis parallels the severity of the disease. C, $\mathrm{J}$ - Densitometric analysis of ET-1 immunofluorescence intensity of human (C) and chicken (J). Each open square $(\square)$ represents the ET-1 value of both $1 \mathrm{hHC}$ and $1 \mathrm{cHC}$; each solid square ( $\mathbf{\square})$ represents the ET-1 value of $1 \mathrm{SSc}$ patient and 1 UCD-200 sample. Our results are expressed as median (range) of the immunofluorescence optical density (arbitrary units). The ET-1 expression of SSC patients was significantly higher than in hHC (ET-1, hHC vS. SSc: $\left.{ }^{* * *} p<0.0001\right)$. Significantly higher ET-1 optical density was observed in each UCD-200 group than in cHC. Furthermore, a significant increase of the optical density was observed in parallel with the severity of comb involvement (ET-1, erythema vs. edema: ${ }^{* *} p=0.0002$; edema vs. necrosis: ${ }^{* *} p$ $=0.0002)$. D, K - Densitometric analysis of the intensity of immunofluorescence staining of human (D) and chicken (K) ETAR. Each open dot (O) represents the ETAR value of both $1 \mathrm{hHC}$ and $1 \mathrm{cHC}$; each solid dot $(\mathbf{0})$ represents the ETAR value of 1 SSc patient and 1 UCD-200 sample respectively. Our results are expressed as median (range) of the immunofluorescence optical density (arbitrary units). The expression of ETAR was significantly higher in SSc patients than in hHC (ETAR, hHC vs. SSc: $\left.{ }^{* \star *} p<0.0001\right)$. Significantly higher ETAR optical density was observed in each UCD-200 group than in CHC. Furthermore, a significant difference of the optical density was observed when any group of UCD-200 samples was compared with another, showing a progressive increase of the optical density in parallel with the severity of comb involvement (ETAR, erythema vs. edema: ${ }^{* *} p<0.0001$; edema vs. necrosis: $\left.{ }^{* *} p=0.0002\right)$. E, L - Densitometric analysis of the intensity of human $(\mathrm{E})$ and chicken $(\mathrm{L}) \mathrm{ETBR}$ immunofluorescence staining . Each open rhombus $(\diamond)$ represents the ETBR value of both $1 \mathrm{hHC}$ and $1 \mathrm{cHC}$; each solid rhombus ( $)$ represents the ETBR value of 1 SSc patient and 1 UCD-200 sample respectively. Our results are expressed as median (range) of the immunofluorescence optical density (arbitrary units). The expression of ETBR was significantly higher in SSc patients than in hHC (ETBR, hHC vs. SSc: $\left.{ }^{* *} p<0.0001\right)$. Significantly higher ETBR optical density was observed in each UCD-200 group than in CHC. Furthermore, a significant difference of the optical density was observed when any group of UCD-200 samples was compared with another, showing a progressive increase of the optical density in parallel with the severity of comb involvement (ETBR, erythema vs. edema: ${ }^{*} p=0.0004$; edema vs. necrosis: $\left.{ }^{*} p=0.0004\right)$ 


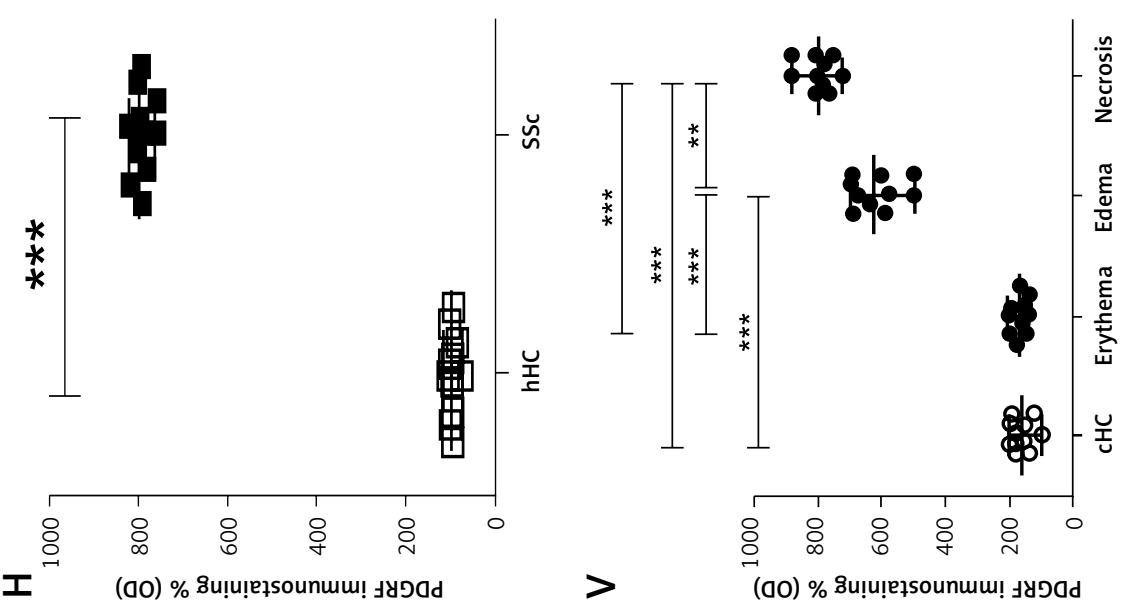

che early phase of human systemic sclerosis

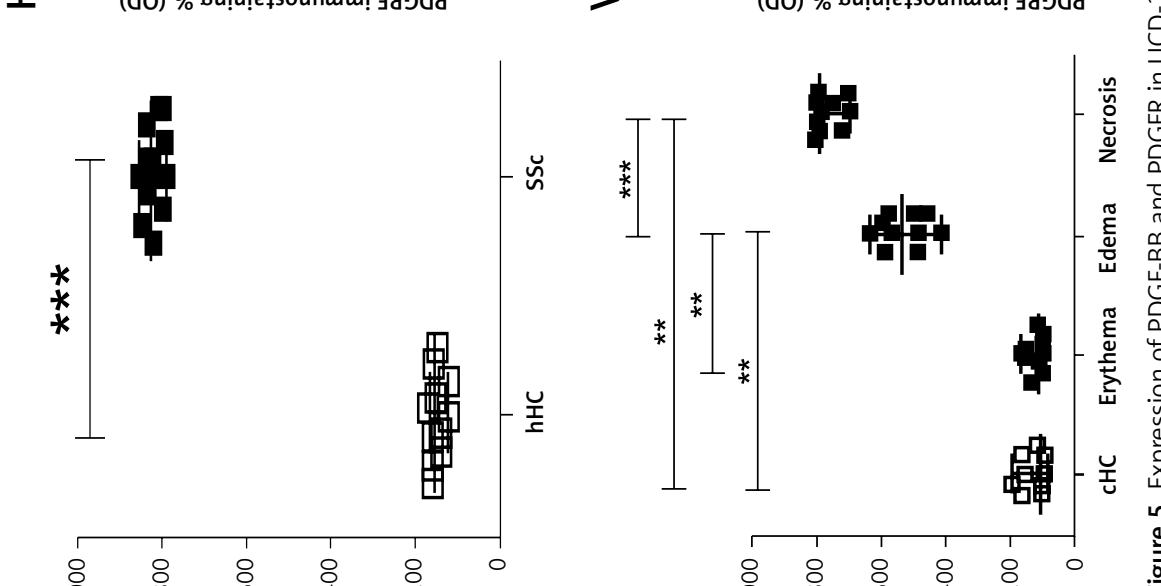

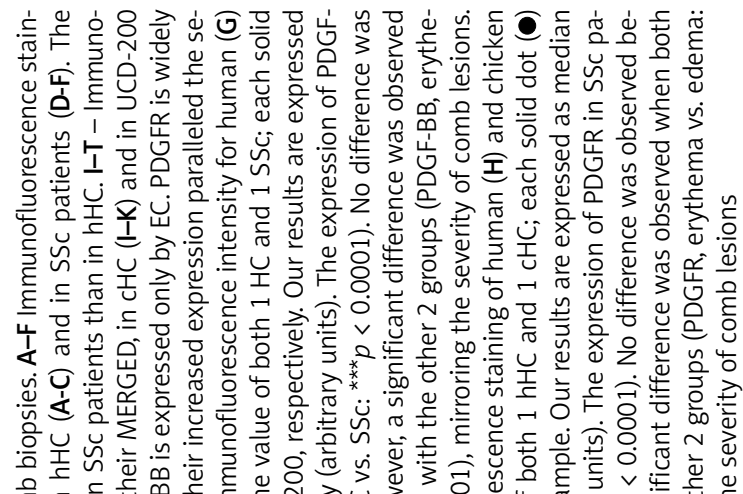

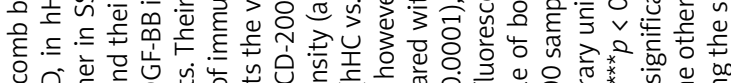

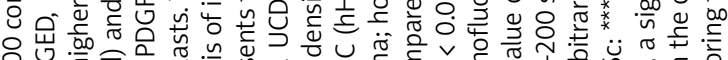

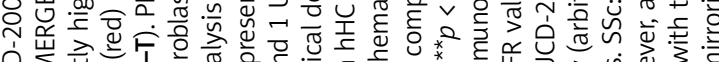

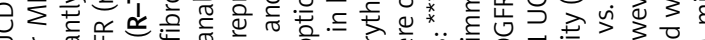

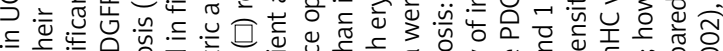

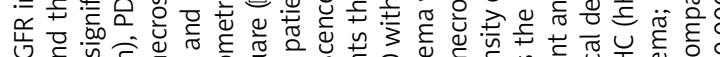

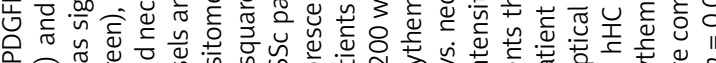

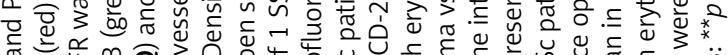

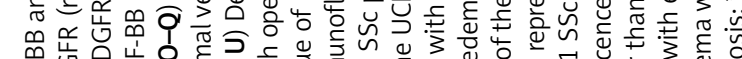

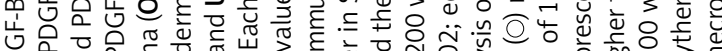

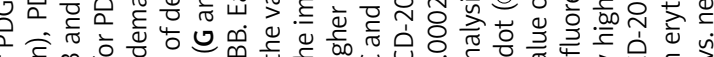

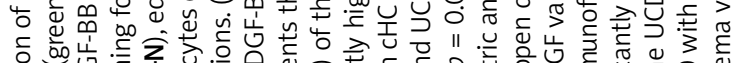

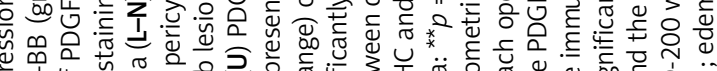

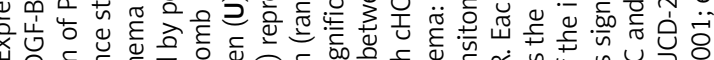
幽믐든

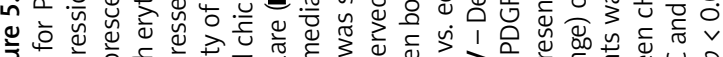

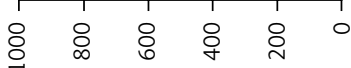

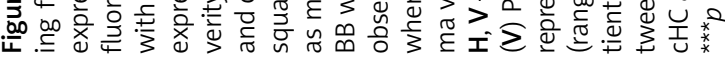

$\circlearrowleft$

(ه0) \% 8u!u!̣ęsounmu! gg--900d

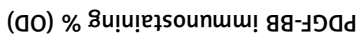
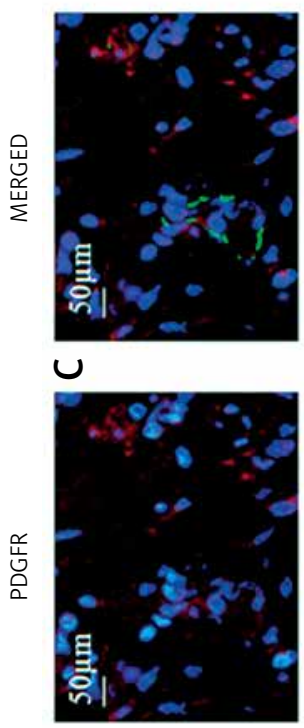

$\infty$

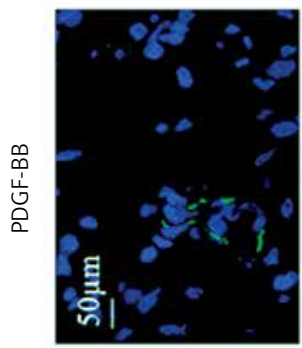

$\varangle$
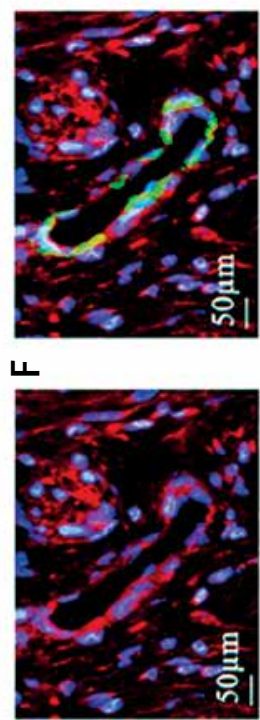

ш

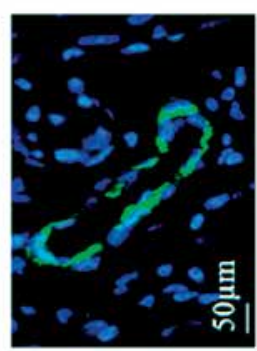

口

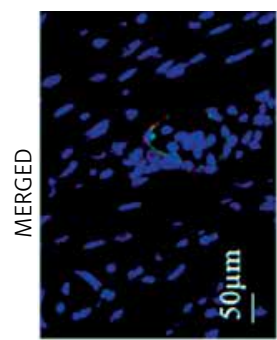

Y

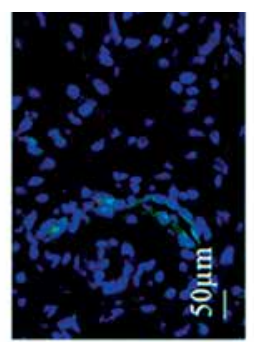

$z$
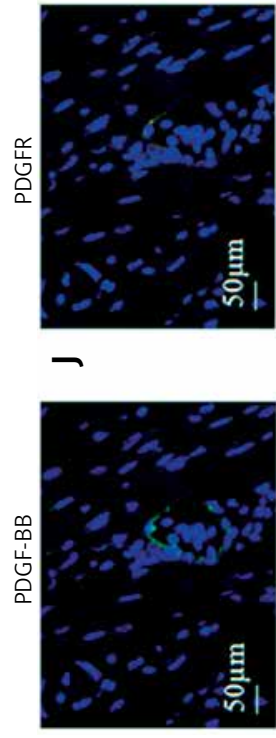

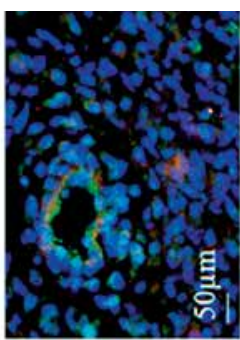

$O^{\prime}$

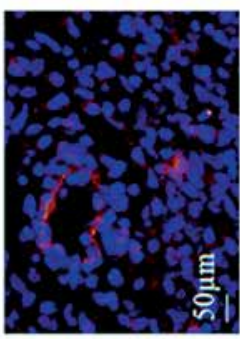

a

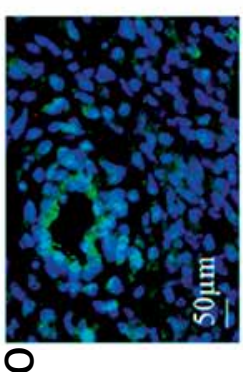

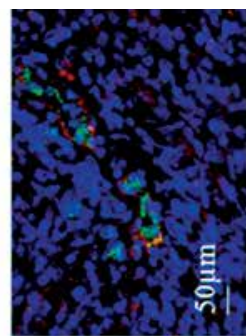

$\vdash$

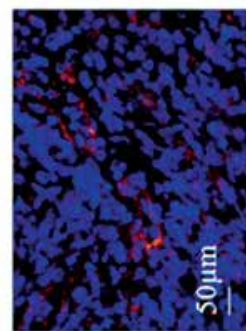

$\sim$

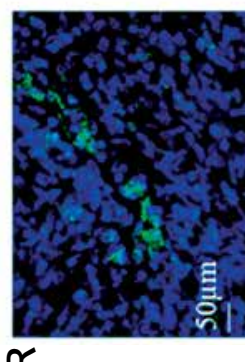



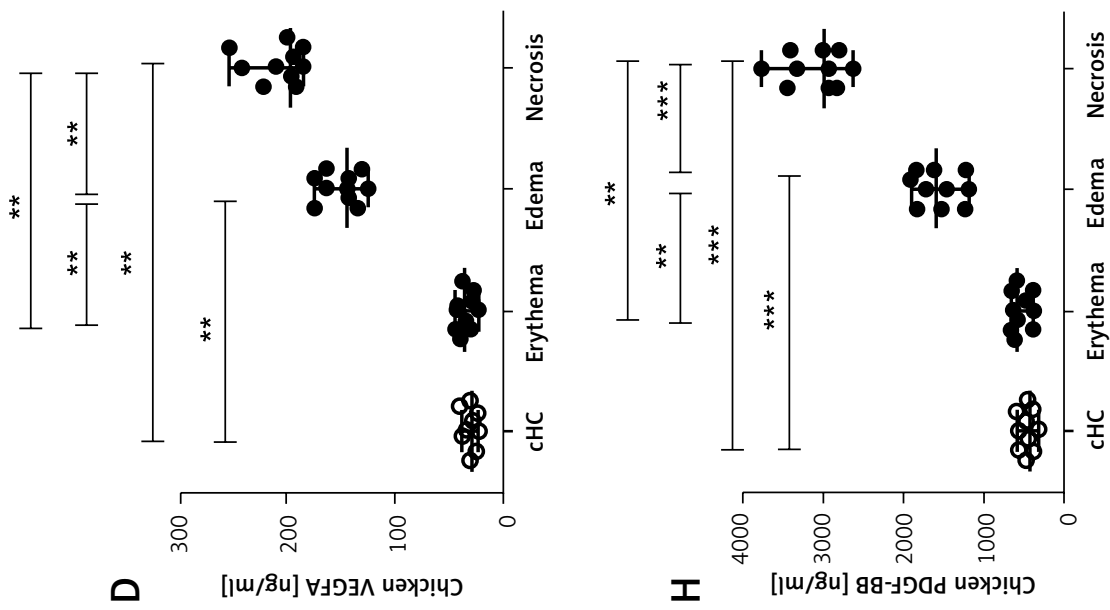

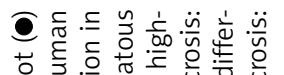

응

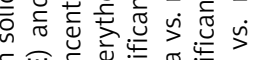

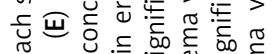

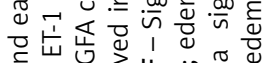

桴

志舟

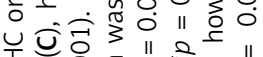

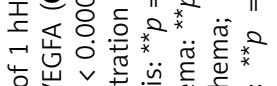

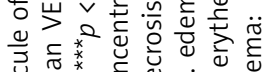

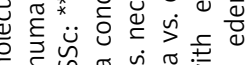

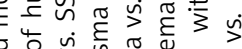

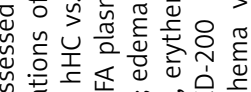

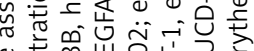
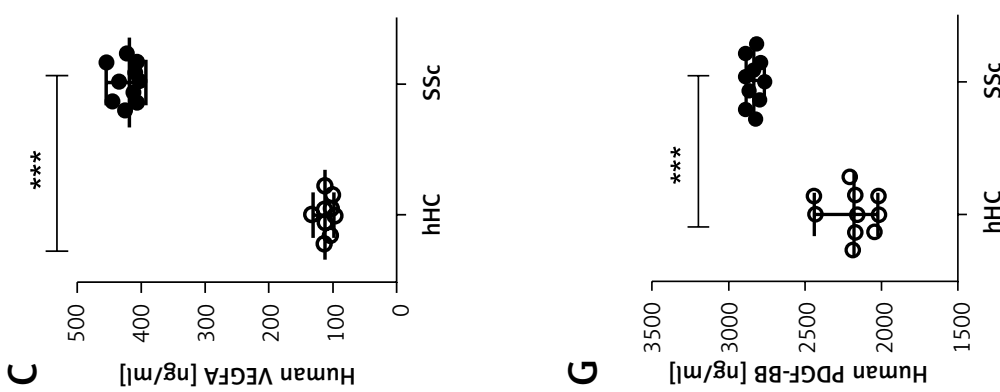

品>出。

。

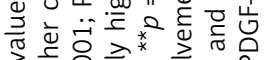

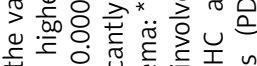

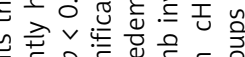

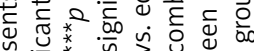

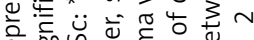

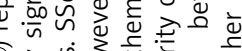

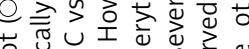

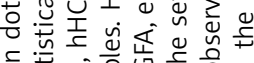

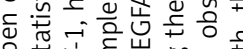

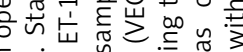

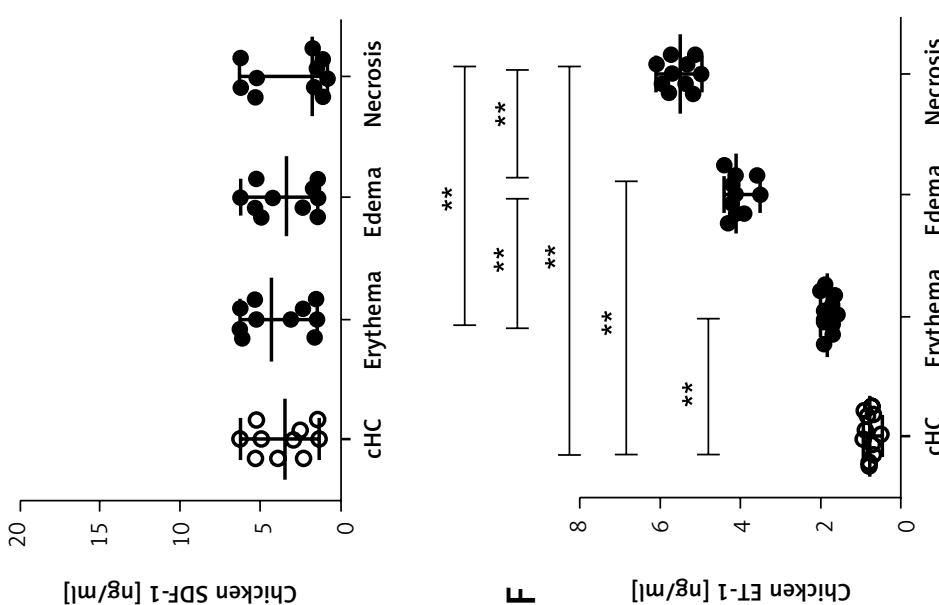

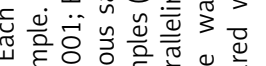

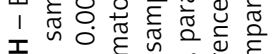

준 8 v

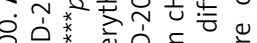

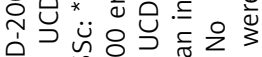

Q

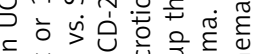

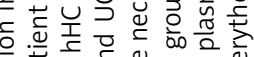

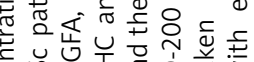

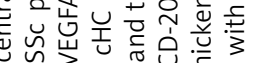

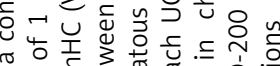

है

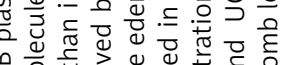

œ

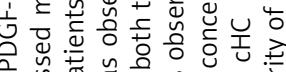

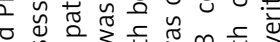

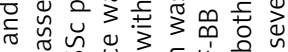

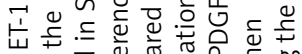

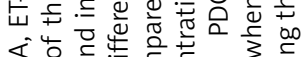

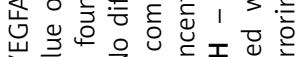

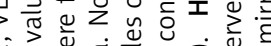

ن̀

ט

ช

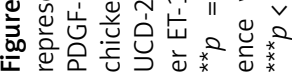

$\varangle \quad$ [|w/8u] [--gas uewn

س $\quad[\mid w / 8 u][-1 \exists$ ueunH 
the combs and not involving internal organs. Our results showed that SDF-1 and CXCR4 were also expressed by fibroblast cells and their intensity of fluorescence was strongly increased in necrotic biopsies. Circulating fibrocytes, expressing CXCR4, have been reported to migrate to sites of injury and differentiate into myofibroblasts, which may be responsible for collagen production in fibrotic tissue [22]. Fibroblasts, releasing SDF-1, actively contribute to endothelial progenitor cell recruitment, for tissue regeneration, and support chronic inflammation by regulating the recruitment of leukocytes [23]. Taken together, the increase of the SDF-1/CXCR4 axis observed in UCD-200 fibroblasts may support the inflammatory process, and finally, fibrosis.

During human SSc, despite severe tissue hypoxia, adapted angiogenesis does not occur. Indeed, defective vascularization contrasts with the elevated levels of VEGFA found in the serum and in the skin of SSc patients throughout the different SSC phases $[24,25]$. VEGFA exerts its effects by binding to VEGFR-1 and VEGFR-2. It was reported that the expression of VEGFR-1 and VEGFR-2 was significantly increased in the skin of SSc patients [26]. VEGFA and VEGFRs are normally expressed by ECs, Ps and fibroblasts, contributing to vessel assembly. During inflammation, inflammatory cells and fibroblasts migrate into the injured site, produce extracellular matrix and express VEGFA [27, 28]. VEGFA, released from ECs and fibroblasts, might contribute to the development and survival of newly formed blood capillaries by promoting both EC and P recruitment [29]. Furthermore, Ps, in contact with EC, produce VEGF that may act in a paracrine manner as a survival and/or stabilizing factor for EC in vessels. On these bases, we reported that VEGFA and VEGFR2 were significantly higher in human SSc skin than in hHC skin. The evidence of increased expression of the VEGFA/VEGFR2/VEGFR1 axis in UCD-200, despite vascular alterations, with significantly reduced vessel density, suggests that the impaired angiogenesis in UCD-200 closely mirrors what has already been reported in human SSc.

Although the trigger mechanisms which are related to the onset of SSc are still largely unknown, it is widely accepted that activated myofibroblasts may promote SSc skin fibrosis by overproduction of collagen-rich extracellular matrix (ECM) [30]. However, the origin of these ECM-producing fibroblasts in SSc has not been conclusively demonstrated [31]. Many different cell types have been proposed, including endothelial cells, mural cells, bone marrow-derived cells, and mesenchymal stem and/or progenitor cells. It is well established that profibrotic pathways can be activated by cytokines, for example TGF- $\beta 1$, PDGF-BB and ET-1, which are likely to be crucially involved in the pathogenesis of SSc.
The TGF- $\beta$ involvement in avian SSc has been studied by Prelog et al. in a previous work, suggesting a possible explanation of the contradictory observations regarding the role of type 2 isoform of TGF- $\beta$ in human SSc [32], showing, in this avian model of disease, that a decrease of TGF- $\beta 2$ synthesis might act as a pro-fibrotic stimulus.

To understand the ET-1 contribution to the avian disease, we assessed in UCD-200 combs the ET-1/ETAR/ETBR expression.

ET-1 has an important role in human SSc, displaying a potent endogenous vasoconstrictor action, which also mediates vascular wall cell proliferation, fibrosis, and inflammation [33]. ET-1 mediates different biological effects through two ET receptors, ETAR and ETBR, both expressed on ECs, Ps and fibroblasts. Altered cellular expression of ET receptors may be associated with different functional responses. Elevated ET-1 expression was observed in skin of patients with early active SSc, associated with increased expression of the ET-1 receptors, at the skin vessels and at the dermal/epidermal junction in SSc skin [34]. Consistent with these observations, increased ET-1 production was observed in SSc fibroblasts, deriving from the affected skin [35]. In SSc-associated fibrotic lung tissue, ETA receptor levels were slightly reduced and ETB receptor levels were slightly elevated, compared with healthy tissue [36]. Confirming these data, we found that levels of ET-1 and its receptors were significantly higher in SSc patients than in hHC. Furthermore, in UCD-200 comb biopsies we found a significant increase of ET-1 and both ETA and ETB receptors. All these molecules showed increased expression in vascular cells and fibroblasts in the early phase of disease, before the onset of fibrosis. We may speculate that in the edematous and necrotic phase of disease, the strong release of ET-1 may be the result of the vascular alterations, resulting in receptor activation. As regards PDGF-BB, it is well known that PDGF is secreted by platelets, monocytes, vascular endothelial cells, and other cell types and binds PDGFR, expressed by fibroblasts and pericytes. A significant increase in expression of PDGFR on Ps within the dermal microvasculature is observed in patients with SSC of recent onset, and fibrotic skin lesions in SSC patients express higher levels of PDGF and PDGF receptors [37-39]. Furthermore, elevated levels of PDGF have been detected in bronchoalveolar lavage of SSc patients. Our results confirmed that PDGF-BB and its receptor were significantly higher in SSc human skin than hHC. Mirroring these results, in UCD-200 we found significantly higher PDGF-BB expression, compared with $\mathrm{CHC}$, and the increased expression of the PDGF-BB/PDGFR axis correlated with severity of lesions, mirroring the evidence reported in human SSc [40]. 
As regards plasma levels of SDF-1, VEGFA, ET-1 and PDGF-BB, we found that the circulating levels of these molecules mirror what has been observed in human SSc. In fact, circulating levels of VEGFA were increased in UCD-200, similarly to SSC patients [24]. At present, both the triggers and the sources of VEGFA in plasma samples of UCD-200 remain to be defined. Furthermore, according to human SSc, our study confirms that, in this avian disease, ET-1 and PDGF-BB sera levels were higher than in $\mathrm{hHC}$ and seemed to increase together with the progression of the disease.

Considering the failure in compensative vasculogenesis of SSc patients, up-regulation of the SDF-1 level could be expected, due to its positive effect on the recruitment, growth and survival of cells from the bone marrow. Surprisingly, the serum concentration of SDF-1 in human SSc was comparable to healthy controls, suggesting that the circulating homeostasis of SDF-1 and CXCR4 is maintained and the decreased level of SDF-1/ CXCR4 axis in the skin of late SSc patients does not influence the serum level of SDF-1 in SSc patients. The discrepancies between the plasma and tissue levels of SDF-1 in UCD-200 mirror those observed in human SSc [41].

In conclusion, this is the first paper reporting the SDF-1, VEGFA, PDGF-BB and ET-1 alterations in UCD-200. These findings strongly confirm that these molecules, which are involved in human SSc, seem to be similarly involved in this avian model of the disease.

The strong molecular similarities between avian and human SSc observed in this study make the UCD-200 chickens an interesting model to identify the early pathogenetic events linking the vascular damage and the fibrotic process during human disease and to perform new translational therapeutic approaches to SSC.

\section{Acknowledgments}

The authors thank Mrs. Federica Sensini for her technical assistance.

This work was supported by FIRA (Fondazione Italiana Ricerca per l'Artrite) 2009 and the Austrian Science Fund (FWF): P23230-B13.

\section{Conflict of interest}

The authors declare no conflict of interest.

\section{References}

1. Trojanowska M. Role of PDGF in fibrotic diseases and systemic sclerosis. Rheumatology 2008; 47: v2-4.

2. Cipriani P, Marrelli A, Di Benedetto P, et al. Sclerodermia mesenchymal stem cells display a different phenotype from healthy controls; implications for regenerative medicine. Angiogenesis 2013; 16: 595-607.
3. Liakouli V, Cipriani P, Marrelli A, Alvaro S, Ruscitti P, Giacomelli R. Angiogenic cytokines and growth factors in systemic sclerosis. Autoimmun Rev 2011; 10: 590-4.

4. Wick G, Andersson L, Hala K, et al. Avian models with spontaneous autoimmune diseases. Adv Immunol 2006; 92: 71-117.

5. Beyer C, Schett G, Distler O, Distler JHW. Animal models of systemic sclerosis: prospects and limitations. Arthritis Rheum 2010; 62: 2831-44.

6. Sgonc R, Wick G. Pro- and anti-fibrotic effects of TGF-beta in scleroderma. Rheumatology (Oxford) 2008; 475: v5-7.

7. Del Galdo F, Matucci-Cerinic M. The search for the perfect animal model discloses the importance of biological targets for the treatment of systemic sclerosis. Ann Rheum Dis 2014; 73: 635-6.

8. van den Hoogen F, Khanna D, Fransen J, et al. 2013 classification criteria for systemic sclerosis: an American College of Rheumatology/European League against Rheumatism Collaborative Initiative. Arthritis Rheum 2013; 65: 2737-47.

9. van den Hoogen F, Khanna D, Fransen J, et al. 2013 classification criteria for systemic sclerosis: an American College of Rheumatology/European League Against Rheumatism Collaborative Initiative. Ann Rheum Dis 2013; 72: 1747-55.

10. Hunzelmann N, Genth E, Krieg T, et al. Registry of the German Network for Systemic Scleroderma. The registry of the German Network for Systemic Scleroderma: frequency of disease subsets and patterns of organ involvement. Rheumatology (Oxford) 2008; 47: 1185-92.

11. Waszczykowska A, Goś R, Waszczykowska E, Dziankowska-Bartkowiak B, Jurowski P. Prevalence of ocular manifestations in systemic sclerosis patients. Arch Med Sci 2013; 9: 1107-13.

12. Sgonc R, Gruschwitz MS, Dietrich H, Recheis H, Gershwin ME, Wick G. Endothelial cell apoptosis is a primary pathogenetic event underlying skin lesions in avian and human scleroderma. J Clin Invest 1996; 98: 785-92.

13. Sgonc R. The vascular perspective of systemic sclerosis: of chickens, mice and men. Int Arch Allergy Immunol 1999; 120: 169-76.

14. Kucia M, Jankowski K, Reca R, et al. CXCR4-SDF-1 signalling, locomotion, chemotaxis and adhesion. J Mol Histol 2004; 35: 233-45.

15. Jo DY, Rafii S, Hamada T, Moore MA. Chemotaxis of primitive hematopoietic cells in response to stroma cell derived factor-1. J Clin Invest 2000; 105: 101-11.

16. Aghi M, Cohen KS, Klein RJ, Scadden DT, Chiocca EA. Tumor stromal-derived factor-1 recruits vascular progenitors to mitotic neovasculature, where micro-environment influences their differentiated phenotypes. Cancer Res 2006; 66: 9054-64.

17. Pablos IL, Amara A, Bouloc A, et al. Stromal-cell derived factor is expressed by dendritic cells and endothelium in human skin. Am J Pathol 1999; 155: 1577-86.

18. Cipriani P, Franca Milia A, Liakouli V, et al. Differential expression of stromal cell-derived factor 1 and its receptor CXCR4 in the skin and endothelial cells of systemic sclerosis patients: pathogenetic implications. Arthritis Rheumatism 2006; 54: 3022-33.

19. Seo J, Kim YO, Jo I. Differential expression of stromal cell-derived factor 1 in human brain microvascular endothelial cells and pericytes involves histone modifications. Biochem Biophys Res Commun 2009; 382: 519-24.

20. Middleton J, Neil S, Wintle J, et al. Transcytosis and surface presentation of IL-8 by venular endothelial cells. Cell 1997; 91: 385-95. 
21. Abe R, Donnelly SC, Peng T et al. Peripheral blood fibrocytes: differentiation pathway and migration to wound sites. J Immunol 2001; 166: 7556-62.

22. Phillips RJ, Burdick MD, Hong K, et al. Circulating fibrocytes traffic to the lungs in response to CXCL12 and mediate fibrosis. J Clin Invest 2004; 114: 438-46.

23. Distler O, Distler JH, Scheid A, et al. Uncontrolled expres sion of vascular endothelial growth factor and its receptors leads to insufficient skin angiogenesis in patients with systemic sclerosis. Circ Res 2004; 95: 109-16.

24. Distler O, Del Rosso A, Giacomelli R, et al. Angiogenic and angiostatic factors in systemic sclerosis: increased levels of vascular endothelial growth factor are a feature of the earliest disease stages and are associated with the absence of fingertip ulcers. Arthritis Res 2002; 4: R11.

25. Ciurzyński M, Bienias P, Irzyk K, et al. Heart diastolic dys function in patients with systemic sclerosis. Arch Med Sci 2014; 10: 445-54

26. Mackiewicz Z, Sukura A, Povilenaité D, et al. Increased but imbalanced expression of VEGF and its receptors has no positive effect on angiogenesis in systemic sclerosis skin. Clin Exp Rheumatol 2002; 20: 641-6.

27. Hagedorn $M$, Balke $M$, Schmidt $A$, et al. VEGF coordinates interaction of pericytes and endothelial cells during vasculogenesis and experimental angiogenesis. Dev Dyn 2004; 230: 23-33.

28. Dąbek J, Głogowska-Ligus J, Bochenek A. Expression of angiogenesis-related genes in right atrial appendages from patients with advanced myocardial ischemia-preliminary results. Arch Med Sci 2013; 9: 540-3.

29. Leroy EC. Connective tissue synthesis by scleroderma skin fibroblasts in cell culture. J Exp Med 1972; 135 1351-62.

30. Vancheeswaran R, Magoulas T, Efrat G, et al. Circulating endothelin-1 levels in systemic sclerosis subsets: a marker of fibrosis or vascular dysfunction? J Rheumatol 1994; 21: 1838-44

31. Rajkumar VS, Sundberg C, Abraham DJ, Rubin K, Black CM. Activation of microvascular pericytes in autoimmune Raynaud's phenomenon and systemic sclerosis. Arthritis Rheum 1999; 42: 930-41.

32. Prelog M, Scheidegger P, Peter S, Gershwin ME, Wick G, Sgonc R. Diminished TGF-beta2 production leads to increased expression of a profibrotic procollagen alpha 2 type I mRNA variant in embryonic fibroblasts of UCD200 chickens, a model for systemic sclerosis. Arthritis Rheum 2005; 52: 1804-11.

33. Cambrey AD, Harrison NK, Dawes KE, et al. Increased levels of endothelin-1 in bronchoalveolar lavage fluid from patients with systemic sclerosis contribute to fibroblast mitogenic activity in vitro. Am J Respir Cell Mol Biol 1994; 11: 439-45.

34. Vancheeswaran R, Azam A, Black C, Dashwood MR. Localization of endothelin-1 and its binding sites in scleroderma skin. J Rheumatol 1994; 21: 1268-76.

35. Kawaguchi Y, Suzuki K, Hara M, et al. Increased endothelin-1 production in fibroblasts derived from patients with systemic sclerosis. Ann Rheum Dis 1994; 53: 506-10

36. Abraham DJ, Vancheeswaran R, Dashwood MR, et al. Increased levels of endothelin-1 and differential endo thelin type $A$ and $B$ receptor expression in scleroderma-associated fibrotic lung disease. Am J Pathol 1997; 151: 831-41.

37. Gay S, Jones Jr RE, Huang GQ, Gay RE. Immunohistologic demonstration of platelet-derived growth factor (PDGF) and sisoncogene expression in scleroderma. J Invest Dermatol 1989; 92: 301-3.

38. Klareskog L, Gustafsson R, Scheynius A, Hallgren R. Increased expression of platelet-derived growth factor type $B$ receptors in the skin of patients with systemic sclerosis. Arthritis Rheum 1990; 33: 1534-41.

39. Cipriani P, Di Benedetto P, Ruscitti P, et al. Impaired endothelium-mesenchymal stem cells cross-talk in systemic sclerosis: a link between vascular and fibrotic features. Arthritis Res Ther 2014; 16: 442.

40. Daoussis D, Tsamandas AC, Liossis SN, et al. B-cell depletion therapy in patients with diffuse systemic sclerosis associates with a significant decrease in PDGFR expression and activation in spindle-like cells in the skin. Arthritis Res Ther 2012; 14: R145.

41. Dziankowska-Bartkowiak B, Gerlicz-Kowalczuk Z, Waszczykowska E. Angiogenin and SDF-1alpha serum concentration in patients with systemic sclerosis in relation to clinical status. Arch Med Sci 2011; 7: 92-6. 